\begin{tabular}{|c|c|}
\hline Title & Classical scale mixtures of Boolean stable laws \\
\hline Author(s) & A rizmendi, Octavio; Hasebe, Takahiro \\
\hline Citation & $\begin{array}{l}\text { Transactions of the A merican Mathematical Society, 368(7), 4873-4905 } \\
\text { https://doi.org/10.1090/tran } 6792\end{array}$ \\
\hline Issue Date & $2016-07$ \\
\hline Doc URL & http:/hdl. handle.net/2115/66350 \\
\hline Rights & $\begin{array}{l}\text { First published in Trans. A mer. Math. Soc. in 368(7) 2016, published by the A merican Mathematical Society. @2015 } \\
\text { A merican Mathematical Society }\end{array}$ \\
\hline Rights(URL) & https://creativecommons.org/icenses/by-nc-nd/2.0/ \\
\hline Type & article (author version) \\
\hline File Information & A rizmendiHasebeTA MS.pdf \\
\hline
\end{tabular}

Instructions for use 


\title{
CLASSICAL SCALE MIXTURES OF BOOLEAN STABLE LAWS
}

\author{
OCTAVIO ARIZMENDI AND TAKAHIRO HASEBE
}

\begin{abstract}
We study Boolean stable laws, $\mathbf{b}_{\alpha, \rho}$, with stability index $\alpha$ and asymmetry parameter $\rho$. We show that the classical scale mixture of $\mathbf{b}_{\alpha, \rho}$ coincides with a free mixture and also a monotone mixture of $\mathbf{b}_{\alpha, \rho}$. For this purpose we define the multiplicative monotone convolution of probability measures, one is supported on the positive real line and the other is arbitrary.

We prove that any scale mixture of $\mathbf{b}_{\alpha, \rho}$ is both classically and freely infinitely divisible for $\alpha \leq 1 / 2$ and also for some $\alpha>1 / 2$. Furthermore, we show the multiplicative infinite divisibility of $\mathbf{b}_{\alpha, 1}$ with respect classical, free and monotone convolutions.

Scale mixtures of Boolean stable laws include some generalized beta distributions of second kind, which turn out to be both classically and freely infinitely divisible. One of them appears as a limit distribution in multiplicative free laws of large numbers studied by Tucci, Haagerup and Möller.

We use a representation of $\mathbf{b}_{\alpha, 1}$ as the free multiplicative convolution of a free Bessel law and a free stable law to prove a conjecture of Hinz and Młotkowski regarding the existence of the free Bessel laws as probability measures. The proof depends on the fact that $\mathbf{b}_{\alpha, 1}$ has free divisibility indicator 0 for $1 / 2<\alpha$.
\end{abstract}

\section{Contents}

1. Introduction

2. Preliminaries 4

2.1. Notations 4

2.2. Additive Convolutions 5

2.3. Multiplicative Convolutions 6

2.4. Free Infinite Divisibility $\quad 7$

2.5. Stable Distributions 9

3. Basic Results 9

3.1. Multiplicative Monotone Convolution: General Case 9

3.2. Transforms and Identities for Stable Laws 11

4. Scale Mixtures of Boolean Stable Laws 13

4.1. Definition and Properties 13

4.2. Connections between Classical, Free and Boolean Stable Laws 16

4.3. Classical and Multiplicative Infinite Divisibility 18

$\begin{array}{ll}\text { 4.4. Free Infinite Divisibility of } \mathcal{B}_{\alpha, \rho} & 19\end{array}$

Date: August 3, 2015.

2010 Mathematics Subject Classification. 46L54, $60 \mathrm{E} 07$.

Key words and phrases. Free convolution, Boolean stable law, infinite divisibility, mixtures, free Bessel law.

T. Hasebe was supported by Marie Curie Actions - International Incoming Fellowships (Project 328112 ICNCP) at University of Franche-Comté and also by Global COE program "Fostering top leaders in mathematics - broadening the core and exploring new ground" at Kyoto university. 
5. Examples 23

5.1. Explicit Densities of Probability Measures in $\mathcal{B}_{1 / 2,1} \quad 23$

5.2. Limit Distributions of Multiplicative Free Laws of Large Numbers 24

5.3. Continuous Boolean Convolution 26

5.4. Probability Measures in $\mathcal{B}_{1, \rho} \backslash \mathcal{I D}(\boxplus) \quad 27$

5.5. Free Jurek Class and $\mathcal{B}_{\alpha, \rho}$

6. Existence of Free Bessel Laws 29

6.1. Free Powers of Free Poisson 29

6.2. Free Bessel Laws 30

References $\quad 31$

\section{Introduction}

In this paper we study different aspects of classical scale mixtures of Boolean stable laws $\mathbf{b}_{\alpha, \rho}$ including classical and free infinite divisibility, unimodality and relation with other distributions such as classical stable laws, free stable laws and free Bessel laws.

We denote respectively by $*, \boxplus$ the classical and free additive convolutions, and by $\circledast, \bigotimes$ the classical and free multiplicative convolutions. An important class of measures in connection with the study of limit laws is the class of infinitely divisible distributions. A probability measure $\mu$ is said to be (classically) infinitely divisible (or ID for short) if, for every natural number $n$, there exists a probability measure $\mu_{n}$ such that

$$
\mu=\underbrace{\mu_{n} * \mu_{n} * \cdots \mu_{n}}_{n \text { times }}
$$

In the same way, in free probability a measure $\mu$ is said to be freely infinitely divisible (or FID for short) if, for every natural number $n$, there exists a probability measure $\mu_{n}$ such that

$$
\mu=\underbrace{\mu_{n} \boxplus \mu_{n} \boxplus \cdots \boxplus \mu_{n}}_{n \text { times }} .
$$

We denote by $\mathcal{I D}(*)$ the class of all ID distributions on $\mathbb{R}$ and by $\mathcal{I D}(\boxplus)$ the class of all FID distributions on $\mathbb{R}$.

The Boolean stable law $\mathbf{b}_{\alpha, \rho}$ appears as the stable distribution for Boolean independence [41]. The positive one $\mathbf{b}_{\alpha, 1}$ is the law of quotient of identically distributed, independent positive $\alpha$-stable random variables. The density is given by

$$
\frac{\frac{1}{\pi} \sin (\alpha \pi) x^{\alpha-1}}{x^{2 \alpha}+2 \cos (\alpha \pi) x^{\alpha}+1}, x>0 .
$$

The authors studied these measures in [2] in relation to classical and free infinite divisibility, proving that the Boolean stable law is FID for $\alpha \leq 1 / 2$ or $1 / 2<\alpha \leq$ $2 / 3,2-1 / \alpha \leq \rho \leq 1 / \alpha-1$. Moreover the positive Boolean stable law for $\alpha \leq 1 / 2$ is both ID and FID. Note that Jedidi and Simon showed that it is HCM, more strongly than ID [30]. The positive Boolean stable law was the first nontrivial continuous family of measures which are ID and FID.

Our main result is in Section 4. We extend the results in [2] to classical scale mixtures of Boolean stable laws, giving a large class of probability measures being ID and FID. 
Theorem 1.1. Let $B_{\alpha, \rho}$ be a random variable following the Boolean stable law $\mathbf{b}_{\alpha, \rho}$, and let $X$ be any nonnegative random variable classically independent of $B_{\alpha, \rho}$. If $\alpha \in(0,1 / 2]$ or if $\alpha \leq 2 / 3, \rho=1 / 2$, then the law of $X B_{\alpha, \rho}$ is in $\mathcal{I D}(\boxplus) \cap \mathcal{I D}(*)$.

The proof is given separately for ID and FID parts. We show in Theorem 4.18 that the law of $X B_{\alpha, \rho}$ is ID if: (a) $\alpha \leq 1 / 2$; (b) $\alpha \leq 1, \rho=1 / 2$. (a), (b) may not be necessary conditions for $X B_{\alpha, \rho}$ being ID. The proof depends on mixtures of exponential distributions for $\alpha \leq 1 / 2$ and mixtures of Cauchy distributions for $\rho=1 / 2$. For the free part, we show in Theorem 4.25 that the law of $X B_{\alpha, \rho}$ is FID for any $X \geq 0$ if and only if: (i) $\alpha \leq 1 / 2$; (ii) $1 / 2<\alpha \leq 2 / 3,2-1 / \alpha \leq \rho \leq 1 / \alpha-1$. The proof is based on complex analysis; we show that the Voiculescu transform has an analytic extension defined in $\mathbb{C}^{+}$taking values in $\mathbb{C}^{-} \cup \mathbb{R}$ (see [16]). When $B_{\alpha, \rho}$ is symmetric or positive, we give a simpler proof by using the identities

$$
\begin{array}{ll}
\mathbf{b}_{\alpha, 1}=\boldsymbol{\pi}^{\bigotimes \frac{1-\alpha}{\alpha}} \otimes \mathbf{f}_{\alpha, 1}, & \alpha \in(0,1], \\
\mathbf{b}_{\alpha, 1 / 2}=\boldsymbol{\pi}^{\bigotimes \frac{2-\alpha}{2 \alpha}} \otimes \operatorname{Sym}\left(\sqrt{\mathbf{f}_{\alpha / 2,1}}\right), & \alpha \in(0,2],
\end{array}
$$

where $\mathbf{f}_{\alpha, \rho}$ is a free stable law and $\boldsymbol{\pi}$ is a free Poisson. See Section 2 for the other notations. We also show the multiplicative infinite divisibility for $\mathbf{b}_{\alpha, 1}$ with respect to classical, free and monotone convolutions.

In Subsections 3.2 and 4.1, we establish a lot of identities involving classical, Boolean and free stable laws, and multiplicative classical $\circledast$, free $\nabla$ and monotone $\circlearrowright$ convolutions. For this purpose, we define the multiplicative monotone convolution of two probability measures, one is supported on $[0, \infty)$ and the other is on $\mathbb{R}$, in Subsection 3.1. The most outstanding result in this context is the following identity:

$$
\mu^{1 / \alpha} \circledast \mathbf{b}_{\alpha, \rho}=\mu^{\bowtie 1 / \alpha} \otimes \mathbf{b}_{\alpha, \rho}, \quad \mathbf{b}_{\alpha, \rho} \text { being positive or symmetric. }
$$

The measure $\mu^{1 / \alpha}$ is the law of $X^{1 / \alpha}$ when $X$ follows the law $\mu$. This identity gives us a direct connection between classical multiplication and free multiplication, and it suggests the importance of $\mathbf{b}_{\alpha, \rho}$. In Subsection 4.2, we compare classical, free and Boolean stable laws and observe similarities between them.

Examples of random variables $X B_{\alpha, \rho}$ as in Theorem 1.1 are provided in Section 5. We give new probability measures which are both ID and FID, including the generalized beta distributions of the second kind with densities

$$
\begin{array}{lr}
c_{\alpha, \beta} \cdot \frac{x^{\alpha-3 / 2}}{\left(x^{\alpha \beta}+1\right)^{1 / \beta}} 1_{(0, \infty)}(x), & \alpha \in(1 / 2,1], \beta \in(0,1 / \alpha], \\
\frac{\beta x^{\beta-1}}{\left(x^{\beta}+1\right)^{2}} 1_{(0, \infty)}(x), & \beta \in(0,1 / 2],
\end{array}
$$

see Proposition 5.1 and Example 5.5. Moreover, these measures are HCM (see [17]). We compute the limit distributions in free multiplicative laws of large numbers $[44,21]$

$$
\lim _{n \rightarrow \infty}\left(\mu^{\otimes n}\right)^{1 / n}
$$

by taking $\mu$ to be the law of $X B_{\alpha, 1}$. The limit distribution is again a scale mixture of Boolean stable laws, but now with stability index $\alpha /(1-\alpha)$. We consider the free Jurek class which is the free analogue of Jurek class [31]. The law of $X B_{\alpha, \rho}$ belongs to the free Jurek class for $\alpha \lesssim 0.42, \rho=1$ and for $\alpha \leq 1 / 2, \rho=1 / 2$.

Free Bessel laws, introduced in Banica et al. [8], are measures $\boldsymbol{\pi}_{s t}=(1-t) \delta_{0}+$ $D_{t}\left(\left(\boldsymbol{\pi}^{\bigotimes s}\right)^{\boxplus 1 / t}\right)$ for $s>0,0<t \leq 1$, where $\boldsymbol{\pi}$ is the free Poisson with mean 1 . Note 
that $\boldsymbol{\pi}_{s t}$ is a probability measure since $\boldsymbol{\pi}$ is $\bigotimes$-infinitely divisible. It is also known that the parameters may be extended to $s \geq 1, t>0$. The question of whether one can extend these parameters for $0<s<1, t>1$ was raised in [8]. Later, from considerations of moments, Hinz and Młotkowski [28] conjectured that $\left(\boldsymbol{\pi}^{\otimes s}\right)^{\boxplus t}$ is not a probability measure for $0<s, t<1$. In the last part of the paper, we give an answer to the conjecture of Hinz and Młotkowski using the representation (1.1) and the free divisibility indicator, and we then settle the question of the existence of free Bessel laws as a corollary.

Theorem 1.2. Let $s, t>0$ and let $\tilde{\boldsymbol{\pi}}_{s t}=\left(\boldsymbol{\pi}^{\otimes s}\right)^{\boxplus t}$. Then $\tilde{\boldsymbol{\pi}}_{s t}$ is a probability measure if and only if $\max (s, t) \geq 1$. In other words, the sequence of Fuss-Narayana polynomials given by

$$
\tilde{m}_{0}(s, t)=1, \quad \tilde{m}_{n}(s, t)=\sum_{k=1}^{n} \frac{t^{k}}{n}\left(\begin{array}{c}
n \\
k-1
\end{array}\right)\left(\begin{array}{c}
n s \\
n-k
\end{array}\right), \quad n \geq 1
$$

is a sequence of moments of a probability measure on $\mathbb{R}$ if and only if $\max (s, t) \geq 1$. In particular, the free Bessel law $\boldsymbol{\pi}_{\text {st }}$ is a probability measure if and only if $(s, t) \in$ $(0, \infty) \times(0, \infty)-(0,1) \times(1, \infty)$.

\section{Preliminaries}

2.1. Notations. We collect basic notations used in this paper.

(1) $\mathbb{N}=\{1,2,3, \ldots\}$ is the set of strictly positive natural numbers.

(2) $\mathcal{P}$ is the set of (Borel) probability measures on $\mathbb{R}$.

(3) $\mathcal{P}_{+}$is the set of probability measures on $\mathbb{R}_{+}=[0, \infty)$.

(4) $\mathcal{P}_{s}$ is the set of symmetric probability measures on $\mathbb{R}$.

(5) For $a \in \mathbb{R}$, we denote by $D_{a} \mu$ the dilation of a probability measure $\mu$, i.e. if a random variable $X$ follows $\mu$, then $D_{a} \mu$ is the law of $a X$.

(6) $\mathbb{C}^{+}, \mathbb{C}^{-}$denote the complex upper half-plane and the lower half-plane, respectively.

(7) For $D \subset \mathbb{C} \backslash\{0\}$, the set $D^{-1}$ denotes the image of $D$ by the map $z \mapsto z^{-1}$.

(8) For $\theta_{1}<\theta_{2}$ such that $\theta_{2}-\theta_{1} \leq 2 \pi, \mathbb{C}_{\left(\theta_{1}, \theta_{2}\right)}$ is the sector $\left\{r e^{i \theta}: r>0, \theta \in\right.$ $\left.\left(\theta_{1}, \theta_{2}\right)\right\}$.

(9) For $\alpha, \beta>0, \Gamma_{\alpha, \beta}$ is the truncated cone $\left\{z \in \mathbb{C}^{+}:|\operatorname{Re}(z)|<\alpha \operatorname{Im}(z),|z|>\beta\right\}$.

(10) For $p \in \mathbb{R}$ and $\mu \in \mathcal{P}_{+}$, let $\mu^{p}$ be the push-forward of $\mu$ by the map $x \mapsto x^{p}$. If $p$ is an integer, we define $\mu^{p}$ for any $\mu \in \mathcal{P}$. If $p \leq 0$, we define $\mu^{p}$ only when $\mu(\{0\})=0$. We may use the notation $\sqrt{\mu}$ instead of $\mu^{1 / 2}$.

(11) For $\mu \in \mathcal{P}_{+}$, the measure $\operatorname{Sym}(\mu)$ is the symmetrization $\frac{1}{2}(\mu(d x)+\mu(-d x))$.

(12) For $z \in \mathbb{C} \backslash\left(-\mathbb{R}_{+}\right)$, arg $z$ is the argument of $z$ taking values in $(-\pi, \pi)$.

(13) For $p \in \mathbb{R}$, the power $z \mapsto z^{p}$ denotes the principal value $|z|^{p} e^{i p \arg z}$ in $\mathbb{C} \backslash$ $\left(-\mathbb{R}_{+}\right)$.

(14) For $\theta_{1}<\theta_{2}$ such that $\theta_{2}-\theta_{1} \leq 2 \pi, \arg _{\left(\theta_{1}, \theta_{2}\right)} z$ denotes the argument of $z \in$ $\mathbb{C}_{\left(\theta_{1}, \theta_{2}\right)}$ taking values in $\left(\theta_{1}, \theta_{2}\right)$.

(15) For $p \in \mathbb{R}$ and $\theta_{1}<\theta_{2}$ such that $\theta_{2}-\theta_{1} \leq 2 \pi$, the power $z \mapsto(z)_{\left(\theta_{1}, \theta_{2}\right)}^{p}$ is defined by $|z|^{p} \exp \left(i p \arg _{\left(\theta_{1}, \theta_{2}\right)} z\right)$ for $z \in \mathbb{C}_{\left(\theta_{1}, \theta_{2}\right)}$. 
2.2. Additive Convolutions. We briefly explain the additive convolutions from non-commutative probability used in this paper. They correspond to notions of independence coming from universal products classified by Muraki [34]: tensor (classical), free, Boolean and monotone independences. We omit monotone convolution since it does not appear in this paper.

2.2.1. Classical Convolution. Let $\mathcal{F}_{\mu}$ be the characteristic function of $\mu \in \mathcal{P}$. Then the classical convolution is characterized by

$$
\mathcal{F}_{\mu_{1} * \mu_{2}}(z)=\mathcal{F}_{\mu_{1}}(z) \mathcal{F}_{\mu_{2}}(z), \quad z \in \mathbb{R}, \mu_{1}, \mu_{2} \in \mathcal{P} .
$$

Classical convolution corresponds to the sum of (tensor) independent random variables.

The moment generating function of $\mu \in \mathcal{P}$ is defined by $\mathcal{M}_{\mu}(z):=\mathcal{F}_{\mu}(z / i)$, $z \in i \mathbb{R}$. When $\mu \in \mathcal{P}_{+}$, the domain of $\mathcal{M}_{\mu}$ extends to $\{z \in \mathbb{C}: \operatorname{Re}(z) \leq 0\}$. For $\mu \in \mathcal{P}$, there exists $a \in(0, \infty]$ such that $\mathcal{M}_{\mu}(z) \neq 0$ in $i(-a, a)$, and then we may define the classical cumulant transform of $\mu \in \mathcal{P}$ by

$$
\mathcal{C}_{\mu}^{*}(z)=\log \left(\mathcal{M}_{\mu}(z)\right), \quad z \in i(-a, a)
$$

such that it is continuous and $\mathcal{C}_{\mu}^{*}(0)=0$. It then follows that for some $c>0$ depending on $\mu_{1}, \mu_{2}$,

$$
\mathcal{C}_{\mu_{1} * \mu_{2}}^{*}(z)=\mathcal{C}_{\mu_{1}}^{*}(z)+\mathcal{C}_{\mu_{2}}^{*}(z), \quad z \in i(-c, c) .
$$

In general, $\mathcal{C}^{*}$ does not characterize the probability measure, that is, there are two distinct $\mu, \nu \in \mathcal{P}$ such that $\mathcal{C}_{\mu}^{*}(z)=\mathcal{C}_{\nu}^{*}(z)$ in some $i(-a, a)$. In particular cases such as $\mu \in \mathcal{I D}(*)$, the characteristic function does not have a zero and hence the classical cumulant transform $\mathcal{C}_{\mu}^{*}$ extends to a continuous function on $i \mathbb{R}$. Then $\mathcal{C}_{\mu}^{*}$ on $i \mathbb{R}$ (or on $-i \mathbb{R}_{+}$) uniquely determines $\mu$.

2.2.2. Free Convolution. Free convolution was defined in [45] for compactly supported probability measures and later extended in [33] for the case of finite variance, and in [16] for the general unbounded case. Let

$$
G_{\mu}(z)=\int_{\mathbb{R}} \frac{\mu(d x)}{z-x}, \quad F_{\mu}(z)=\frac{1}{G_{\mu}(z)}, \quad z \in \mathbb{C} \backslash \mathbb{R}
$$

be the Cauchy transform and the reciprocal Cauchy transform (or Ftransform) of $\mu \in \mathcal{P}$, respectively. It was proved in Bercovici and Voiculescu [16] that there exist $\alpha, \beta, \alpha^{\prime}, \beta^{\prime}>0$ such that $F_{\mu}$ is univalent in $\Gamma_{\alpha^{\prime}, \beta^{\prime}}$ and $F_{\mu}\left(\Gamma_{\alpha^{\prime}, \beta^{\prime}}\right) \supset$ $\Gamma_{\alpha, \beta}$. Hence the left compositional inverse $F_{\mu}^{-1}$ may be defined in $\Gamma_{\alpha, \beta}$. The Voiculescu transform of $\mu$ is then defined by $\phi_{\mu}(z)=F_{\mu}^{-1}(z)-z$ on the region $\Gamma_{\alpha, \beta}$ where $F_{\mu}^{-1}$ is defined. Moreover, the free cumulant transform (see [9]) is a variant of $\phi_{\mu}$ defined as

$$
\mathcal{C}_{\mu}^{\boxplus}(z)=z \phi_{\mu}\left(\frac{1}{z}\right)=z F_{\mu}^{-1}\left(\frac{1}{z}\right)-1, \quad z \in\left(\Gamma_{\alpha, \beta}\right)^{-1} .
$$

The free convolution of two probability measures $\mu_{1}, \mu_{2}$ on $\mathbb{R}$ is the probability measure $\mu_{1} \boxplus \mu_{2}$ on $\mathbb{R}$ such that

$$
\phi_{\mu_{1} \boxplus \mu_{2}}(z)=\phi_{\mu_{1}}(z)+\phi_{\mu_{2}}(z)
$$

in a common domain $\Gamma_{\gamma, \delta}$ which is contained in the intersection of the domains of $\phi_{\mu_{1}}, \phi_{\mu_{2}}$ and $\phi_{\mu_{1} \boxplus \mu_{2}}$. Free convolution corresponds to the sum of free random variables [16]. 
For any $t \geq 1$ and any $\mu \in \mathcal{P}$, there exists a measure $\mu^{\boxplus t} \in \mathcal{P}$ which satisfies $\phi_{\mu}^{\boxplus t}(z)=t \phi_{\mu}(z)$ in a common domain [37].

2.2.3. Boolean Convolution. The Boolean convolution [41] of two probability measures $\mu_{1}, \mu_{2}$ on $\mathbb{R}$ is defined as the probability measure $\mu_{1} \uplus \mu_{2}$ on $\mathbb{R}$ such that

$$
\eta_{\mu_{1} \uplus \mu_{2}}(z)=\eta_{\mu_{1}}(z)+\eta_{\mu_{2}}(z), z \in \mathbb{C}^{-},
$$

where the $\eta$-transform (or Boolean cumulant transform) is defined by

$$
\eta_{\mu}(z)=1-z F_{\mu}\left(\frac{1}{z}\right), \quad z \in \mathbb{C}^{-} .
$$

Boolean convolution corresponds to the sum of Boolean independent random variables. Such an operator-theoretic model was constructed in [41] for bounded operators and in [20] for unbounded operators.

For any $t \geq 0$ and any $\mu \in \mathcal{P}$, there exists a measure $\mu^{\uplus t} \in \mathcal{P}$ which satisfies $\eta_{\mu^{\uplus t}}(z)=t \eta_{\mu}(z)$ in $\mathbb{C}^{-}[41]$.

\subsection{Multiplicative Convolutions.}

2.3.1. Multiplicative Classical Convolution. The multiplicative classical convolution $\mu_{1} \circledast \mu_{2}$ of $\mu_{1}, \mu_{2} \in \mathcal{P}$ is defined by

$$
\int_{\mathbb{R}} f(x)(\mu \circledast \nu)(d x)=\int_{\mathbb{R}} f(x y) \mu(d x) \nu(d y)
$$

for any bounded continuous function $f$ on $\mathbb{R}$. The measure $\mu_{1} \circledast \mu_{2}$ corresponds to the distribution of $X Y$, where $X$ and $Y$ are independent random variables with distributions $\mu_{1}$ and $\mu_{2}$, respectively.

2.3.2. Multiplicative Free Convolution. For probability measures $\mu_{1} \in \mathcal{P}_{+}, \mu_{2} \in \mathcal{P}$, the multiplicative free convolution $\mu_{1} \otimes \mu_{2} \in \mathcal{P}$ is defined as the distribution of $\sqrt{X} Y \sqrt{X}$, where $X \geq 0$ and $Y$ are free random variables with distributions $\mu_{1}$ and $\mu_{2}$, respectively. Multiplicative free convolution was introduced in [47] for compactly supported probability measures, and then extended in [16] for non compactly supported probability measures.

Suppose that $\delta_{0} \neq \mu \in \mathcal{P}_{+}$(resp. $\left.\delta_{0} \neq \mu \in \mathcal{P}_{s}\right)$. The function $\eta_{\mu}$ is univalent around $(-\infty, 0)$ (resp. $i(-\infty, 0))$ taking values in a neighborhood of the interval $\left(1-(\mu(\{0\}))^{-1}, 0\right)$ (we understand that $(\mu(\{0\}))^{-1}=\infty$ if $\left.\mu(\{0\})=0\right)$, so that one may define the compositional inverse $\eta_{\mu}^{-1}$ and then the $\Sigma$-transform

$$
\Sigma_{\mu}(z):=\frac{\eta_{\mu}^{-1}(z)}{z}, \quad z \in\left(1-(\mu(\{0\}))^{-1}, 0\right) .
$$

Multiplicative free convolution $\otimes$ is characterized by the multiplication of $\Sigma$-transforms:

$$
\Sigma_{\mu_{1} \otimes \mu_{2}}(z)=\Sigma_{\mu_{1}}(z) \Sigma_{\mu_{2}}(z), \quad \mu_{1} \in \mathcal{P}_{+}, \mu_{2} \in \mathcal{P}_{+} \text {or } \mu_{2} \in \mathcal{P}_{s}
$$

in the common interval $(-\beta, 0)$, provided $\mu_{1} \neq \delta_{0} \neq \mu_{2}$. The case $\mu_{1}, \mu_{2} \in \mathcal{P}_{+}$ was proved in [16] and the case $\mu_{1} \in \mathcal{P}_{+}, \mu_{2} \in \mathcal{P}_{s}$ was proved in [6]. When $\mu_{1} \in$ $\mathcal{P}_{+}, \mu_{2} \in \mathcal{P}$ and they have compact supports, (2.2) was proved in a neighborhood of 0 in [47] and [38]. In the most general case $\mu_{1} \in \mathcal{P}_{+}$and $\mu_{2} \in \mathcal{P}$, it is still an open problem to define an appropriate $S$-transform $S_{\mu_{2}}$ and to prove (2.2). 
Instead of the $\Sigma$-transform, often used to calculate multiplicative free convolution is the $S$-transform:

$$
S_{\mu}(z)=\Sigma_{\mu}\left(\frac{z}{1+z}\right), \quad z \in(-1+\mu(\{0\}), 0) .
$$

If $\mu \in \mathcal{P}_{+}$, then a convolution power $\mu^{\bigotimes t} \in \mathcal{P}_{+}$, satisfying $\Sigma_{\mu} \otimes_{t}(z)=\left(\Sigma_{\mu}(z)\right)^{t}$, is well defined for any $t \geq 1$ ([11]). A probability measure $\mu \in \mathcal{P}_{+}$is said to be $\bigotimes$-infinitely divisible if for any $n \in \mathbb{N}$, there is $\mu_{n}$ on $\mathbb{R}_{+}$such that $\mu=\mu_{n}^{\bigotimes n}=$ $\mu_{n} \otimes \cdots \otimes \mu_{n}$.

For $\mu \in \mathcal{P}_{+}, \nu \in \mathcal{P}$, the identity

$$
D_{1 / t}\left(\mu^{\boxplus t} \otimes \nu^{\boxplus t}\right)=(\mu \otimes \nu)^{\boxplus t}, \quad t \geq 1
$$

was proved in [13, Proposition 3.5].

Using the $S$-transform, it was proved in [6] that, for $\mu \in \mathcal{P}_{+}$and $\nu \in \mathcal{P}_{s}$, the following relation holds:

$$
(\mu \otimes \nu)^{2}=\mu \otimes \mu \otimes \nu^{2} .
$$

Assume $\mu \in \mathcal{P}_{+}$and $\mu(\{0\})=0$. The following formula is known [22, Proposition 3.13]:

$$
S_{\mu^{-1}}(z)=\frac{1}{S_{\mu}(-z-1)}, \quad z \in(-1,0) .
$$

\subsection{Free Infinite Divisibility.}

2.4.1. Characterization, Lévy-Khintchine Representation. Recall that a probability measure $\mu$ is ID if and only if its classical cumulant transform $\mathcal{C}_{\mu}^{*}$ has the LévyKhintchine representation (see e.g. [39])

$$
\mathcal{C}_{\mu}^{*}(z)=\eta z+\frac{1}{2} a z^{2}+\int_{\mathbb{R}}\left(e^{z t}-1-z t 1_{[-1,1]}(t)\right) \nu(d t), \quad z \in i \mathbb{R},
$$

where $\eta \in \mathbb{R}, a \geq 0$ and $\nu$ is a Lévy measure on $\mathbb{R}$, that is, $\int_{\mathbb{R}} \min \left(1, t^{2}\right) \nu(d t)<\infty$ and $\nu(\{0\})=0$. If this representation exists, the triplet $(\eta, a, \nu)$ is unique and is called the classical characteristic triplet of $\mu$.

A FID measure has a free analogue of the Lévy-Khintchine representation.

Theorem 2.1 (Voiculescu [46], Maassen [33], Bercovici \& Voiculescu [16], Barndorff-Nielsen \& Thorbjørnsen [9]). For a probability measure $\mu$ on $\mathbb{R}$, the following are equivalent.

(1) $\mu$ belongs to $\mathcal{I D}(\boxplus)$.

(2) $-\phi_{\mu}$ extends to a Pick function, i.e. an analytic map of $\mathbb{C}^{+}$into $\mathbb{C}^{+} \cup \mathbb{R}$.

(3) For any $t>0$, there exists a probability measure $\mu^{\boxplus t}$ with the property $\phi_{\mu^{\boxplus t}}(z)=$ $t \phi_{\mu}(z)$.

(4) A probability measure $\mu$ on $\mathbb{R}$ is FID if and only if there are $\eta_{\mu} \in \mathbb{R}, a_{\mu} \geq 0$ and a Lévy measure $\nu_{\mu}$ on $\mathbb{R}$ such that

$$
\mathcal{C}_{\mu}^{\boxplus}(z)=\eta_{\mu} z+a_{\mu} z^{2}+\int_{\mathbb{R}}\left(\frac{1}{1-z t}-1-t z 1_{[-1,1]}(t)\right) \nu_{\mu}(d t), \quad z \in \mathbb{C}^{-} .
$$

The triplet $\left(\eta_{\mu}, a_{\mu}, \nu_{\mu}\right)$ is unique and is called the free characteristic triplet of $\mu$ and $\nu_{\mu}$ is called the Lévy measure of $\mu$. 
An important FID distribution in this paper is the free Poisson law $\boldsymbol{\pi}$, also known as the Marchenko-Pastur law, with free characteristic triplet $\left(1,0, \delta_{1}\right)$ and density

$$
\frac{1}{2 \pi} \sqrt{\frac{4-x}{x}} \mathrm{~d} x, \quad 0<x<4 .
$$

The free Poisson distribution $\boldsymbol{\pi}$ is infinitely divisible both with respect to $\bigotimes$ and $\boxplus$.

2.4.2. Compound Free Poisson Distribution. Suppose that $\sigma \in \mathcal{I D}(\boxplus)$ does not have a semicircular component $\left(a_{\sigma}=0\right)$ and that the Lévy measure $\nu_{\sigma}$ in (2.8) satisfies $\int_{\mathbb{R}_{+}} \min (1, t) \nu_{\sigma}(d t)<\infty$. Then the Lévy-Khintchine representation reduces to

$$
\mathcal{C}_{\sigma}^{\boxplus}(z)=\eta_{\sigma}^{\prime} z+\int_{\mathbb{R}}\left(\frac{1}{1-z t}-1\right) \nu_{\sigma}(d t), \quad z \in \mathbb{C}^{-},
$$

where $\eta_{\sigma}^{\prime} \in \mathbb{R}$. The measure $\sigma$ is called the compound free Poisson distribution ([40]) with rate $\lambda$ and jump distribution $\rho$ if the drift term $\eta_{\sigma}^{\prime}$ is zero and the Lévy measure $\nu_{\sigma}$ is $\lambda \rho$ for some $\lambda>0$ and a probability measure $\rho$ on $\mathbb{R}$. To clarify these parameters, we denote $\sigma=\boldsymbol{\pi}(\lambda, \rho)$.

Remark 2.2. (1) The Marchenko-Pastur law $\boldsymbol{\pi}$ is a compound free Poisson with rate 1 and jump distribution $\delta_{1}$.

(2) For any $\nu \in \mathcal{P}$, the compound free Poisson $\boldsymbol{\pi}(1, \nu)$ coincides with the free multiplication $\pi \otimes \nu([36])$.

2.4.3. Free Divisibility Indicator. A one-parameter family of maps $\left\{\mathbb{B}_{t}\right\}_{t \geq 0}$ on $\mathcal{P}$, introduced by Belinschi and Nica [13], is defined by

$$
\mathbb{B}_{t}(\mu)=\left(\mu^{\boxplus(1+t)}\right)^{\uplus \frac{1}{1+t}} .
$$

The family $\left\{\mathbb{B}_{t}\right\}_{t \geq 0}$ is a composition semigroup and, moreover, each map $\mathbb{B}_{t}$ is a homomorphism regarding multiplicative free convolution: $\mathbb{B}_{t}(\mu \otimes \nu)=\mathbb{B}_{t}(\mu) \otimes \mathbb{B}_{t}(\nu)$ for probability measures $\mu \in \mathcal{P}_{+}, \nu \in \mathcal{P}$.

Let $\phi(\mu)$ denote the free divisibility indicator defined by

$$
\phi(\mu):=\sup \left\{t \geq 0: \mu \in \mathbb{B}_{t}(\mathcal{P})\right\},
$$

which has another expression [4]

$$
\phi(\mu)=\sup \left\{t \geq 0: \mu^{\uplus t} \in \mathcal{I D}(\boxplus)\right\} .
$$

For any $\mu \in \mathcal{P}$ and $0 \leq s \leq \phi(\mu)$, Belinschi and Nica proved that a probability measure $\nu_{s}$ uniquely exists such that $\mathbb{B}_{s}\left(\nu_{s}\right)=\mu$. Therefore, the definition of $\mathbb{B}_{t}(\mu)$ may be extended for $0 \geq t \geq-\phi(\mu)$ by setting $\mathbb{B}_{t}(\mu)=\nu_{-t}$. The indicator $\phi(\mu)$ satisfies the following properties [13].

Theorem 2.3. (1) $\mu^{\boxplus t}$ exists if and only if $\phi(\mu) \geq 1-t$.

(2) $\mu$ is FID if and only if $\phi(\mu) \geq 1$.

(3) $\phi\left(\mathbb{B}_{t}(\mu)\right)$ can be calculated as

$$
\phi\left(\mathbb{B}_{t}(\mu)\right)=\phi(\mu)+t, \quad t \geq-\phi(\mu) .
$$

More information on $\mathbb{B}_{t}(\mu)$ and $\phi(\mu)$ is found in $[13,4,29]$. 
2.5. Stable Distributions. Let $\mathfrak{A}$ be the set of admissible parameters:

$$
\mathfrak{A}=\{(\alpha, \rho): \alpha \in(0,1], \rho \in[0,1]\} \cup\left\{(\alpha, \rho): \alpha \in(1,2], \rho \in\left[1-\alpha^{-1}, \alpha^{-1}\right]\right\} .
$$

Definition 2.4. Assume that $(\alpha, \rho)$ is admissible. The classical $\mathbf{n}_{\alpha, \rho}$ (see e.g. [39]), Boolean $\mathbf{b}_{\alpha, \rho}[41]$, free $\mathbf{f}_{\alpha, \rho}[16,15]$ and monotone $\mathbf{m}_{\alpha, \rho}[24,48]$ strictly stable distributions are defined, respectively, by their classical cumulant, $\eta$, free cumulant and $F$ transforms as follows:

$$
\begin{array}{lr}
\mathcal{C}_{\mathbf{n}_{\alpha, \rho}}^{*}(z)=-\left(e^{i \rho \pi} z\right)^{\alpha}, & z \in i(-\infty, 0) ; \\
\eta_{\mathbf{b}_{\alpha, \rho}}(z)=-\left(e^{i \rho \pi} z\right)^{\alpha}, & z \in \mathbb{C}^{-} ; \\
\mathcal{C}_{\mathbf{f}_{\alpha, \rho}}^{\boxplus}(z)=-\left(e^{i \rho \pi} z\right)^{\alpha}, & z \in \mathbb{C}^{-} ; \\
F_{\mathbf{m}_{\alpha, \rho}}(z)=\left(z^{\alpha}+e^{i \rho \alpha \pi}\right)_{(0,2 \pi)}^{1 / \alpha}, & z \in \mathbb{C}^{+} .
\end{array}
$$

The parameters $\alpha, \rho$ are called the stability index and asymmetry parameter.

Remark 2.5. This parametrization follows [25] (except that we include $\alpha=1$ too) and is different from [15] to respect the correspondence with the classical stable distributions [49].

Note that

$$
\mathbf{n}_{1, \rho}=\mathbf{b}_{1, \rho}=\mathbf{f}_{1, \rho}=\mathbf{m}_{1, \rho}, \quad \rho \in[0,1]
$$

and it is the Cauchy distribution $\mathbf{c}_{\rho}$ with density

$$
\frac{1}{\pi} \cdot \frac{\sin \pi \rho}{(x+\cos \pi \rho)^{2}+\sin ^{2} \pi \rho}
$$

with the convention $\mathbf{c}_{0}=\delta_{-1}$ and $\mathbf{c}_{1}=\delta_{1}$.

The probability density functions of the Boolean (and monotone) stable laws are described in [26]. When $\alpha \leq 1$ or when $\alpha>1,1-1 / \alpha<\rho<1 / \alpha$, the Boolean stable law $\mathbf{b}_{\alpha, \rho}$ is absolutely continuous with respect to the Lebesgue measure and the density is given by

$$
p_{\alpha, \rho}^{+}(x) 1_{(0, \infty)}(x)+p_{\alpha, \rho}^{-}(x) 1_{(-\infty, 0)}(x),
$$

where

$$
\begin{aligned}
& p_{\alpha, \rho}^{+}(x)=\frac{\sin (\pi \rho \alpha)}{\pi} \cdot \frac{x^{\alpha-1}}{x^{2 \alpha}+2 x^{\alpha} \cos (\pi \rho \alpha)+1}, \\
& p_{\alpha, \rho}^{-}(x)=\frac{\sin (\pi(1-\rho) \alpha)}{\pi} \cdot \frac{|x|^{\alpha-1}}{|x|^{2 \alpha}+2|x|^{\alpha} \cos (\pi(1-\rho) \alpha)+1} .
\end{aligned}
$$

For $\alpha \in[1,2]$ and $\rho=1-1 / \alpha, 1 / \alpha$, the measure $\mathbf{b}_{\alpha, \rho}$ has one or two atoms.

\section{BAsic Results}

3.1. Multiplicative Monotone Convolution: General Case. The multiplicative monotone convolution of probability measures $\mu_{1}, \mu_{2} \in \mathcal{P}_{+}$is defined as the probability measure $\mu_{1} \circlearrowright \mu_{2} \in \mathcal{P}_{+}$such that

$$
\eta_{\mu_{1} \circlearrowright \mu_{2}}(z)=\eta_{\mu_{1}}\left(\eta_{\mu_{2}}(z)\right), z \in \mathbb{C}^{+} .
$$

Multiplicative monotone convolution corresponds to the operator $\sqrt{X} Y \sqrt{Y}$ (not $\sqrt{Y} X \sqrt{Y}$ ) when $X-1$ and $Y$ are monotone independent random variables [20] and $X, Y \geq 0$. Compactly supported measures $\mu_{1}, \mu_{2} \in \mathcal{P}_{+}$were considered in [14] and measures $\mu_{1}, \mu_{2} \in \mathcal{P}_{+}$with unbounded supports were considered in [20]. 
From the operator model, it is natural to try to define multiplicative monotone convolution for arbitrary $\mu_{1} \in \mathcal{P}_{+}, \mu_{2} \in \mathcal{P}$. Actually the above operator model still works for the general case $\mu_{1} \in \mathcal{P}_{+}, \mu_{2} \in \mathcal{P}$ with a slight modification of proofs.

We will define multiplicative monotone convolution in this general case in terms of complex analysis. For later use, we extract from Belinschi and Bercovici [11] the following characterization of the $\eta$-transform for $\mu \in \mathcal{P}_{+}$.

Proposition 3.1. Let $\delta_{0} \neq \mu \in \mathcal{P}_{+}$. The $\eta$-transform $\eta_{\mu}: \mathbb{C} \backslash \mathbb{R}_{+} \rightarrow \mathbb{C}$ satisfies the following.

(i) $\eta_{\mu}\left(\mathbb{C}^{-}\right) \subset \mathbb{C}^{-}$and $\eta_{\mu}\left(\mathbb{C} \backslash \mathbb{R}_{+}\right) \subset \mathbb{C} \backslash \mathbb{R}_{+}$.

(ii) $\eta_{\mu}(\bar{z})=\overline{\eta_{\mu}(z)}$ for $\mathbb{C} \backslash \mathbb{R}_{+}$.

(iii) $\arg \left(\eta_{\mu}(z)\right) \in(-\pi, \arg z]$ for any $z \in \mathbb{C}^{-}$.

(iv) $\eta_{\mu}(z) \rightarrow 0$ as $z \rightarrow 0$ non tangentially to $\mathbb{R}_{+}$. More precisely, for any $\alpha \in(0, \pi)$ we have

$$
\lim _{z \rightarrow 0, z \in \mathbb{C}_{(-2 \pi+\alpha,-\alpha)}} \eta_{\mu}(z)=0 .
$$

Conversely, if an analytic map $\eta: \mathbb{C} \backslash \mathbb{R}_{+} \rightarrow \mathbb{C}$ satisfies the conditions (i) - (iv), then there exists a probability measure $\delta_{0} \neq \mu \in \mathcal{P}_{+}$such that $\eta=\eta_{\mu}$.

We characterize the $\eta$-transform of a general $\mu \in \mathcal{P}$.

Proposition 3.2. Let $\mu \neq \delta_{0}$ be a probability measure on $\mathbb{R}$. Then the $\eta$-transform $\eta_{\mu}: \mathbb{C}^{-} \rightarrow \mathbb{C}$ is analytic and satisfies the following.

(1) $\eta_{\mu}\left(\mathbb{C}^{-}\right) \subset \mathbb{C} \backslash \mathbb{R}_{+}$.

(2) $\arg z-\pi \leq \arg _{(-2 \pi, 0)}\left(\eta_{\mu}(z)\right) \leq \arg z$ for $z \in \mathbb{C}^{-}$.

(3) $\eta_{\mu}(z) \rightarrow 0$ as $z \rightarrow 0, z \in \mathbb{C}^{-}$non tangentially to $\mathbb{R}$.

Conversely, if an analytic map $\eta: \mathbb{C}^{-} \rightarrow \mathbb{C}$ satisfies the above conditions (1), (2),

(3), then there exists a probability measure $\mu \neq \delta_{0}$ on $\mathbb{R}$ such that $\eta=\eta_{\mu}$.

Remark 3.3. The condition (3) may be replaced by the following simple one:

$$
\lim _{y \uparrow 0} \eta_{\mu}(i y)=0 \text {. }
$$

For our purpose the condition (3) is more useful.

Proof. We have the formula (2.1), and so

$$
\eta(1 / z)=\frac{z-F_{\mu}(z)}{z}
$$

If $\eta\left(1 / z_{0}\right)=c \geq 0$ for some $z_{0} \in \mathbb{C}^{+}$, then $F_{\mu}\left(z_{0}\right)=(1-c) z_{0}$. If moreover $c>0$, then this contradicts the fact that $\operatorname{Im}\left(F_{\mu}(z)\right) \geq \operatorname{Im}(z)$ for $z \in \mathbb{C}^{+}$. If $c=0$, then $F_{\mu}\left(z_{0}\right)=z_{0}$, which is possible only when $\mu=\delta_{0}$, a contradiction. Hence we get (1).

We have $1 / z-F_{\mu}(1 / z) \in \mathbb{C}^{-} \cup \mathbb{R} \backslash\{0\}$ for $z \in \mathbb{C}^{-}$, and hence the condition (2) follows from the identity $\eta(z)=z\left(1 / z-F_{\mu}(1 / z)\right)$.

Since $z-F_{\mu}(z)=o(|z|) \in \mathbb{C}^{-}$as $z \rightarrow \infty, z \in \mathbb{C}^{-}$non tangentially to $\mathbb{R}$ (see $[16])$, we get (3).

Conversely, suppose an analytic map $\eta: \mathbb{C}^{-} \rightarrow \mathbb{C}$ satisfies (1), (2), (3). From (2), the function $z \eta(1 / z)$ maps $\mathbb{C}^{+}$analytically into $\mathbb{C}^{-} \cup \mathbb{R}$. Hence it has a NevanlinnaPick representation

$$
z \eta(1 / z)=-a z+b-\int_{\mathbb{R}} \frac{1+x z}{x-z} \tau(d x), \quad z \in \mathbb{C}^{-}
$$


for some $a \geq 0, b \in \mathbb{R}$ and a nonnegative finite measure $\tau$. Hence

$$
\eta(z)=-a+b z+\int_{\mathbb{R}} \frac{z(x+z)}{1-x z} \tau(d x)=-a+o(1)
$$

as $z \rightarrow 0, z \in \mathbb{C}^{-}$non tangentially to $\mathbb{R}$. From (3) it follows that $a=0$. From [16, Proposition 5.2], there exists $\mu \in \mathcal{P}$ such that $F_{\mu}(z)=z-z \eta(1 / z)$ and hence $\eta=\eta_{\mu}$. The condition (1) implies that $\eta \neq 0$ and hence $\mu \neq \delta_{0}$.

Now we can give a complex analytic definition of $\mu_{1} \circlearrowright \mu_{2}$.

Theorem 3.4. Let $\mu_{1} \in \mathcal{P}_{+}$and $\mu_{2} \in \mathcal{P}$. There exists a probability measure $\mu \in \mathcal{P}$ such that $\eta_{\mu}(z)=\eta_{\mu_{1}}\left(\eta_{\mu_{2}}(z)\right)$ for $z \in \mathbb{C}^{-}$. We denote $\mu=\mu_{1} \circlearrowright \mu_{2}$.

Proof. Proposition 3.1(i) for $\eta_{\mu_{1}}$ and Proposition 3.2(1) for $\eta_{\mu_{2}}$ imply Proposition $3.2(1)$ for $\eta_{\mu_{1}} \circ \eta_{\mu_{2}}$.

Take any $z \in \mathbb{C}^{-}$. Then we have

$$
\eta_{\mu_{2}}(z) \in \mathbb{C}^{-} \Rightarrow\left\{\begin{array}{l}
\arg _{(-2 \pi, 0)}\left(\eta_{\mu_{1}}\left(\eta_{\mu_{2}}(z)\right)\right) \leq \arg _{(-2 \pi, 0)}\left(\eta_{\mu_{2}}(z)\right) \leq \arg (z) \\
\arg _{(-2 \pi, 0)}\left(\eta_{\mu_{1}}\left(\eta_{\mu_{2}}(z)\right)\right)+\pi>0>\arg (z)
\end{array}\right.
$$

where Proposition 3.1(iii) and Proposition 3.2(2) are used on the first line and Proposition 3.1(i) is used on the second. On the other hand, we have:

$$
\begin{aligned}
\eta_{\mu_{2}}(z) & \in \mathbb{C}^{+} \cup(-\infty, 0) \\
\Rightarrow & \left\{\begin{array}{l}
\arg _{(-2 \pi, 0)}\left(\eta_{\mu_{1}}\left(\eta_{\mu_{2}}(z)\right)\right) \leq-\pi<\arg (z), \\
\arg _{(-2 \pi, 0)}\left(\eta_{\mu_{1}}\left(\eta_{\mu_{2}}(z)\right)\right)+\pi \geq \arg (-2 \pi, 0)
\end{array}\left(\eta_{\mu_{2}}(z)\right)+\pi>\arg (z),\right.
\end{aligned}
$$

where Proposition 3.1(i) is used on the first line and Proposition 3.1(iii), (ii) and Proposition 3.2(2) are used on the second. From (3.4) and (3.5), Proposition 3.2(2) holds for $\eta_{\mu_{1}} \circ \eta_{\mu_{2}}$.

Finally, Proposition 3.1(iv) for $\eta_{\mu_{1}}$ and Proposition 3.2(3) for $\eta_{\mu_{2}}$ imply Proposition 3.2(3) for $\eta_{\mu_{1}} \circ \eta_{\mu_{2}}$. Note here that for any $\alpha \in(0, \pi / 2)$, if $z \rightarrow 0, z \in$ $\mathbb{C}_{(-\pi+\alpha,-\alpha)}$, then $\eta_{\mu_{2}}(z) \in \mathbb{C}_{(-2 \pi+\alpha,-\alpha)}$ from Proposition 3.2(2), and hence $\eta_{\mu_{2}}(z) \rightarrow$ 0 non tangentially to $\mathbb{R}_{+}$.

3.2. Transforms and Identities for Stable Laws. The following relations will be often used. 
Proposition $3.5\left(S, \Sigma, \eta\right.$-transforms of $\left.\mathbf{b}_{\alpha, \rho}, \mathbf{f}_{\alpha, \rho}, \mathbf{m}_{\alpha, \rho}, \boldsymbol{\pi}\right)$.

$$
\Sigma_{\mathbf{b}_{\alpha, \rho}}(z)=-e^{-i \rho \pi}(-z)^{\frac{1-\alpha}{\alpha}}, \quad z<0,(\alpha, \rho) \in \mathfrak{A}, \rho \in\{0,1 / 2,1\},
$$

$$
S_{\mathbf{b}_{\alpha, \rho}}(z)=-e^{-i \rho \pi}\left(-\frac{z}{1+z}\right)^{\frac{1-\alpha}{\alpha}}, \quad z \in(-1,0),(\alpha, \rho) \in \mathfrak{A}, \rho \in\{0,1 / 2,1\},
$$

$$
\Sigma_{\mathbf{f}_{\alpha, \rho}}(z)=-e^{-i \rho \pi}\left(\frac{-z}{1-z}\right)^{\frac{1-\alpha}{\alpha}}, \quad z<0,(\alpha, \rho) \in \mathfrak{A}, \rho \in\{0,1 / 2,1\},
$$

$$
S_{\mathbf{f}_{\alpha, \rho}}(z)=-e^{-i \rho \pi}(-z)^{\frac{1-\alpha}{\alpha}}, \quad z \in(-1,0),(\alpha, \rho) \in \mathfrak{A}, \rho \in\{0,1 / 2,1\},
$$

$$
\eta_{\mathbf{m}_{\alpha, \rho}}(z)=1-\left(\left(e^{i \rho \pi} z\right)^{\alpha}+1\right)^{1 / \alpha}, \quad z \in \mathbb{C}^{-},(\alpha, \rho) \in \mathfrak{A},
$$

$$
\Sigma_{\mathbf{m}_{\alpha, \rho}}(z)=-e^{-i \rho \pi} \frac{\left((1-z)^{\alpha}-1\right)^{1 / \alpha}}{-z}, \quad z<0,(\alpha, \rho) \in \mathfrak{A}, \rho \in\{0,1 / 2,1\},
$$

$$
\Sigma_{\boldsymbol{\pi}}(z)=1-z, \quad z<0,
$$

$$
S_{\boldsymbol{\pi}}(z)=\frac{1}{1+z}, \quad z \in(-1,0) .
$$

A direct computation of densities implies the following.

Lemma 3.6. Let $\alpha \in(0,2]$. Then

$$
\left(\mathbf{b}_{\alpha, 1 / 2}\right)^{2}=\mathbf{b}_{\alpha / 2,1} \text {. }
$$

Using the $\Sigma$ - or $S$-transform, we are able to show the following.

Proposition 3.7. (1) Let $\alpha \leq 1$ and $t>0$. Then

$$
\left(\mathbf{b}_{\alpha, 1}\right)^{\bigotimes t}=\mathbf{b}_{\frac{\alpha}{t(1-\alpha)+\alpha}, 1} .
$$

In particular, we have $\left(\mathbf{b}_{1 / 2,1}\right)^{\otimes t}=\mathbf{b}_{1 /(1+t), 1}, t>0$.

(2) Let $\alpha \leq 1$ and $t>0$. Then

$$
\left(\mathbf{b}_{\alpha, 1}\right)^{\circlearrowright t}=\mathbf{b}_{\alpha^{t}, 1},
$$

that is, $\mathbf{b}_{\alpha^{s}, 1} \circlearrowright \mathbf{b}_{\alpha^{t}, 1}=\mathbf{b}_{\alpha^{s+t}, 1}, s, t>0$.

(3) We have the representation

$$
\mathbf{b}_{\alpha, \rho}=\pi^{\bigotimes \frac{1-\alpha}{\alpha}} \otimes \mathbf{f}_{\alpha, \rho}, \quad \alpha \in(0,1], \rho \in\{0,1 / 2,1\} .
$$

(4) The symmetric Boolean stable law $\mathbf{b}_{\alpha, 1 / 2}$ has the representation

$$
\mathbf{b}_{\alpha, 1 / 2}=\boldsymbol{\pi}^{\bigotimes \frac{2-\alpha}{2 \alpha}} \otimes \operatorname{Sym}\left(\sqrt{\mathbf{f}_{\alpha / 2,1}}\right), \quad \alpha \in(0,2] .
$$


Proof. (1) Note that $\frac{\alpha}{t(1-\alpha)+\alpha} \leq 1$. The assertion follows from (3.6) and the identity

$$
\frac{(1-\alpha) t}{\alpha}=\frac{1-\frac{\alpha}{t(1-\alpha)+\alpha}}{\frac{\alpha}{t(1-\alpha)+\alpha}} .
$$

(2) is a consequence of (2.13).

(3) From (3.9), (3.13) and (3.7), we get

$$
\begin{aligned}
S_{\boldsymbol{\pi}^{\otimes \frac{1-\alpha}{\alpha}} \otimes \mathbf{f}_{\alpha, \rho}}(z) & =S_{\mathbf{f}_{\alpha, \rho}}(z) S_{\boldsymbol{\pi}^{\boxplus \frac{1-\alpha}{\alpha}}}(z)=-e^{-i \rho \pi}(-z)^{\frac{1-\alpha}{\alpha}} \frac{1}{(1+z)^{\frac{1-\alpha}{\alpha}}} \\
& =-e^{-i \rho \pi}\left(-\frac{z}{1+z}\right)^{\frac{1-\alpha}{\alpha}}=S_{\mathbf{b}_{\alpha, \rho}}(z), \quad z \in(-1,0) .
\end{aligned}
$$

(4) From (3.14), (3.17) and (2.5), we have the representation

$\left(\mathbf{b}_{\alpha, 1 / 2}\right)^{2}=\mathbf{b}_{\alpha / 2,1}=\pi^{\bigotimes \frac{1-\alpha / 2}{\alpha / 2}} \nabla \mathbf{f}_{\alpha / 2,1}=\pi^{\bigotimes \frac{2-\alpha}{2 \alpha}} \nabla \pi^{\bigotimes \frac{2-\alpha}{2 \alpha}} \nabla \mathbf{f}_{\alpha / 2,1}=\left(\boldsymbol{\pi}^{\bigotimes \frac{2-\alpha}{2 \alpha}} \nabla \operatorname{Sym}\left(\sqrt{\mathbf{f}_{\alpha / 2,1}}\right)\right)^{2}$.

This means that

$$
\mathbf{b}_{\alpha, 1 / 2}=\boldsymbol{\pi}^{\otimes \frac{2-\alpha}{2 \alpha}} \otimes \operatorname{Sym}\left(\sqrt{\mathbf{f}_{\alpha / 2,1}}\right)
$$

\section{Scale Mixtures of Boolean Stable Laws}

In this, the main section of the paper, we find identities between the classical scale mixtures, free mixtures and monotone mixtures of Boolean stable laws. We then consider the classical and free infinite divisibility of scale mixtures of Boolean stable laws.

\subsection{Definition and Properties.}

Definition 4.1. Assume that $(\alpha, \rho)$ is admissible and $\mu \in \mathcal{P}_{+}$.

(1) The measure $\mu \circledast \mathbf{b}_{\alpha, \rho}$ is called a scale mixture (or a classical scale mixture) of $\mathbf{b}_{\alpha, \rho}$.

(2) The measure $\mu \nabla \mathbf{b}_{\alpha, \rho}$ is called a free mixture of $\mathbf{b}_{\alpha, \rho}$.

(3) The measure $\mu \circlearrowright \mathbf{b}_{\alpha, \rho}$ is called a monotone mixture of $\mathbf{b}_{\alpha, \rho}$.

(4) Let $\mathcal{B}_{\alpha, \rho}:=\left\{\nu \circledast \mathbf{b}_{\alpha, \rho}: \nu \in \mathcal{P}_{+}\right\}$be the set of scale mixtures of $\mathbf{b}_{\alpha, \rho}$.

For $\alpha \in(0,1], \rho \in[0,1]$ and $\mu \in \mathcal{P}_{+}$, the scale mixture $\mu \circledast \mathbf{b}_{\alpha, \rho}$ is described as follows:

$\mu \circledast \mathbf{b}_{\alpha, \rho}=\mu(\{0\}) \delta_{0}+(1-\mu(\{0\}))\left(p_{\mu, \alpha, \rho}^{+}(x) 1_{(0, \infty)}(x)+p_{\mu, \alpha, \rho}^{-}(x) 1_{(-\infty, 0)}(x)\right) d x$,

where

$$
\begin{aligned}
& p_{\mu, \alpha, \rho}^{+}(x)=\frac{\sin (\pi \rho \alpha)}{\pi} \int_{(0, \infty)} \frac{x^{\alpha-1} t^{\alpha}}{x^{2 \alpha}+2(x t)^{\alpha} \cos (\pi \rho \alpha)+t^{2 \alpha}} \mu(d t) \\
& p_{\mu, \alpha, \rho}^{-}(x)=\frac{\sin (\pi(1-\rho) \alpha)}{\pi} \int_{(0, \infty)} \frac{|x|^{\alpha-1} t^{\alpha}}{|x|^{2 \alpha}+2|x t|^{\alpha} \cos (\pi(1-\rho) \alpha)+t^{2 \alpha}} \mu(d t)
\end{aligned}
$$

Remark 4.2. Note that the set $\mathcal{B}_{1, \rho}$ coincides with the scale mixtures of the Cauchy distribution $\mathbf{c}_{\rho}$ with Cauchy transform $G_{\mathbf{c}_{\rho}}(z)=\frac{1}{z+e^{i \rho \pi}}$. Since $\mathbf{b}_{1,1}=\delta_{1}$, the set $\mathcal{B}_{1,1}$ coincides with $\mathcal{P}_{+}$. 
A key for proving the results in this section is the following formulas for the different transforms of scale mixtures of Boolean stable laws.

Proposition 4.3. For any admissible pair $(\alpha, \rho)$ and $\mu \in \mathcal{P}_{+}$, the following formulas hold.

$$
\begin{array}{ll}
G_{\mu^{1 / \alpha} \circledast \mathbf{b}_{\alpha, \rho}}(z)=-\frac{1}{z}\left(e^{-i \rho \pi} z\right)^{\alpha} G_{\mu}\left(-\left(e^{-i \rho \pi} z\right)^{\alpha}\right), & z \in \mathbb{C}^{+}, \\
\eta_{\mu^{1 / \alpha} \circledast \mathbf{b}_{\alpha, \rho}}(z)=\eta_{\mu}\left(-\left(e^{i \rho \pi} z\right)^{\alpha}\right), & z \in \mathbb{C}^{-} .
\end{array}
$$

Proof. Let $X, B_{\alpha, \rho}$ be classical independent random variables following the laws $\mu, \mathbf{b}_{\alpha, \rho}$ respectively. Then

$$
\begin{aligned}
G_{\mu \circledast \mathbf{b}_{\alpha, \rho}}(z) & =G_{X B_{\alpha, \rho}}(z)=\mathbb{E}\left[\frac{1}{z-X B_{\alpha, \rho}}\right]=\mathbb{E}\left[\frac{1 / X}{z / X-B_{\alpha, \rho}}\right] \\
& =\mathbb{E}\left[\frac{1}{X} G_{B_{\alpha, \rho}}\left(\frac{z}{X}\right)\right]=\mathbb{E}\left[\frac{1}{z+e^{i \alpha \rho \pi} X^{\alpha} z^{1-\alpha}}\right] \\
& =z^{\alpha-1} \mathbb{E}\left[\frac{1}{z^{\alpha}+e^{i \alpha \rho \pi} X^{\alpha}}\right]=-e^{-i \alpha \rho \pi} z^{\alpha-1} \mathbb{E}\left[\frac{1}{-e^{-i \alpha \rho \pi} z^{\alpha}-X^{\alpha}}\right] \\
& =-e^{-i \rho \pi}\left(e^{-i \rho \pi} z\right)^{\alpha-1} G_{\mu^{\alpha}}\left(-\left(e^{-i \rho \pi} z\right)^{\alpha}\right) .
\end{aligned}
$$

By replacing $\mu$ by $\mu^{1 / \alpha}$, we obtain (4.4). The equality (4.5) follows from (4.4) and $(2.1)$.

In particular, for $\rho=1$, we have explicit formulas for the Cauchy transform and related transforms of $\mu \circledast \mathbf{b}_{\alpha, 1}$.

Corollary 4.4. For $\alpha \in(0,1], \mu \in \mathcal{P}_{+}$, the following formulas hold.

$$
\begin{array}{ll}
G_{\mu^{1 / \alpha} \circledast \mathbf{b}_{\alpha, 1}}(z)=(-z)^{\alpha-1} G_{\mu}\left(-(-z)^{\alpha}\right), & z<0, \\
\eta_{\mu^{1 / \alpha} \circledast \mathbf{b}_{\alpha, 1}}(z)=\eta_{\mu}\left(-(-z)^{\alpha}\right), & z<0 .
\end{array}
$$

Now we show an important formula saying that a scale mixture of $\mathbf{b}_{\alpha, \rho}$ is also a free mixture, and moreover is a monotone mixture.

Theorem 4.5. For any $\mu, \nu \in \mathcal{P}_{+}$, the following relations hold:

$$
\begin{array}{ll}
\mu^{1 / \alpha} \circledast \mathbf{b}_{\alpha, \rho}=\mu^{\bigotimes 1 / \alpha} \otimes \mathbf{b}_{\alpha, \rho}, & \alpha \in(0,1], \rho \in\{0,1 / 2,1\} ; \\
\mu^{1 / \alpha} \circledast \mathbf{b}_{\alpha, \rho}=\mu \circlearrowright \mathbf{b}_{\alpha, \rho}, & (\alpha, \rho) \in \mathfrak{A} .
\end{array}
$$

Remark 4.6. The identity (4.9) is valid for $\alpha>1, \rho=1 / 2$ if $\mu^{\bigotimes 1 / \alpha}$ exists in $\mathcal{P}_{+}$.

Proof. We first show (4.9). (4.5) implies $\eta_{\mu^{1 / \alpha} \circledast \mathbf{b}_{\alpha, \rho}}^{-1}(z)=e^{-i \rho \pi}\left(-\eta_{\mu}^{-1}(z)\right)^{1 / \alpha}$, so that

$$
\begin{aligned}
\Sigma_{\mu^{1 / \alpha} \circledast \mathbf{b}_{\alpha, \rho}}(z) & =\frac{e^{-i \rho \pi}\left(-\eta_{\mu}^{-1}(z)\right)^{1 / \alpha}}{z}=\left(-e^{-i \rho \pi}\right) \frac{\left(-z \Sigma_{\mu}(z)\right)^{1 / \alpha}}{-z} \\
& =\left(\Sigma_{\mu}(z)\right)^{1 / \alpha}\left(-e^{-i \rho \pi}\right)(-z)^{\frac{1-\alpha}{\alpha}}=\Sigma_{\mu^{\boxplus 1 / \alpha}}(z) \Sigma_{\mathbf{b}_{\alpha, \rho}}(z)
\end{aligned}
$$

for $z \in(-c, 0)$ where $\Sigma_{\mu}(z)$ is defined. In the last equality, the formula (3.6) was used. (4.10) follows from (4.5) and (2.13). 
Corollary 4.7. For any probability measures $\mu, \nu \in \mathcal{P}_{+}$, the following relations hold:

$$
\begin{array}{lll}
(4.11) & \left(\mu^{1 / \alpha} \circledast \mathbf{b}_{\alpha, 1}\right) \otimes\left(\nu^{1 / \alpha} \circledast \mathbf{b}_{\alpha, 1}\right)=\left(\mu \otimes \nu \otimes \mathbf{b}_{\frac{1}{2-\alpha}, 1}\right)^{1 / \alpha} \circledast \mathbf{b}_{\alpha, 1}, & \alpha \in(0,1] ; \\
(4.12) & \left(\mu^{1 / \alpha} \circledast \mathbf{b}_{\alpha, \rho}\right) \uplus\left(\nu^{1 / \alpha} \circledast \mathbf{b}_{\alpha, 1}\right)=(\mu \uplus \nu)^{1 / \alpha} \circledast \mathbf{b}_{\alpha, \rho}, & (\alpha, \rho) \in \mathfrak{A} ; \\
(4.13) \quad \mu \circlearrowright\left(\nu^{1 / \alpha} \circledast \mathbf{b}_{\alpha, \rho}\right)=(\mu \circlearrowright \nu)^{1 / \alpha} \circledast \mathbf{b}_{\alpha, \rho}, & (\alpha, \rho) \in \mathfrak{A} .
\end{array}
$$

Proof. (4.11) follows from (4.9) and the relation $\mathbf{b}_{\alpha, 1}=\left(\mathbf{b}_{\frac{1}{2-\alpha}, 1}\right)^{\otimes 1 / \alpha}$.

(4.12) follows from (4.5).

(4.13) follows from the computation

$$
\eta_{\mu \circlearrowright\left(\nu^{1 / \alpha} \circledast \mathbf{b}_{\alpha, \rho}\right)}(z)=\eta_{\mu}\left(\eta_{\nu^{1 / \alpha} \circledast \mathbf{b}_{\alpha, \rho}}(z)\right)=\eta_{\mu}\left(\eta_{\nu}\left(-\left(e^{i \rho \pi} z\right)^{\alpha}\right)\right) .
$$

A particular case of Proposition 4.3 yields a relation between Boolean stable laws with different parameters.

Proposition 4.8. The following relation holds for Boolean stable laws:

$$
\left(\mathbf{b}_{\beta, 1}\right)^{1 / \alpha} \circledast \mathbf{b}_{\alpha, \rho}=\mathbf{b}_{\alpha \beta, \rho}, \quad(\alpha, \rho) \in \mathfrak{A}, \beta \in(0,1] .
$$

Proof. This is an easy comparison of $\eta$-transforms:

$$
\eta_{\left(\mathbf{b}_{\beta, 1}\right)^{1 / \alpha} \circledast \mathbf{b}_{\alpha, \rho}}(z)=\eta_{\mathbf{b}_{\beta, 1}}\left(-\left(e^{i \rho \pi} z\right)^{\alpha}\right)=-\left(e^{i \rho \pi} z\right)^{\alpha \beta}=\eta_{\mathbf{b}_{\alpha \beta, \rho}}(z),
$$

where we used (4.5) on the first equality and $\eta_{\mathbf{b}_{\beta, 1}}(z)=-(-z)^{\beta}$ on the second.

From the previous theorems we can derive closure properties of Boolean mixtures.

Proposition 4.9. (1) For $(\alpha, \rho) \in \mathfrak{A}$, the set $\mathcal{B}_{\alpha, \rho}$ is closed with respect to $\uplus$.

(2) Let $(\alpha, \rho) \in \mathfrak{A}$. If $\sigma \in \mathcal{P}_{+}$and $\tau \in \mathcal{B}_{\alpha, \rho}$, then $\sigma \circledast \tau, \sigma \circlearrowright \tau \in \mathcal{B}_{\alpha, \rho}$.

(3) Let $\alpha \leq 1$. The set $\mathcal{B}_{\alpha, 1}$ is closed with respect to $\circledast, \bigotimes, \uplus, \circlearrowright$.

(4) Let $\alpha \leq 1$. If $\beta \in[\alpha, 1]$ and $\mu \in \mathcal{B}_{\alpha, 1}$, then $\mu^{\beta} \in \mathcal{B}_{\alpha / \beta, 1}$.

(5) Let $\alpha \in(0,1]$ and $t \geq 1$. If $\mu \in \mathcal{B}_{\alpha, 1}$, then $\mu^{\bigotimes t} \in \mathcal{B}_{\frac{\alpha}{t(1-\alpha)+\alpha}}, 1$.

(6) Let $\alpha \leq 1$. If $\tau \in \mathcal{B}_{\alpha, 1}$ and $\tau(\{0\})=0$, then $\tau^{-1} \in \mathcal{B}_{\alpha, 1}$.

Proof. (1) follows from (4.12).

(2) The assertion for $\circledast$ follows by definition. The assertion for $\circlearrowright$ follows from (4.13).

(3) The assertions for $\uplus, \circlearrowright, \circledast$ are included in (1) and (2). The assertion for $\otimes$ follows from (4.11).

(4) From Proposition 4.8 we have $\mathbf{b}_{\beta, 1} \circledast\left(\mathbf{b}_{\alpha / \beta, 1}\right)^{1 / \beta}=\mathbf{b}_{\alpha, 1}$. Taking $\beta$ powers we get $\left(\mathbf{b}_{\beta, 1}\right)^{\beta} \circledast \mathbf{b}_{\alpha / \beta, 1}=\left(\mathbf{b}_{\alpha, 1}\right)^{\beta}$, implying that $\left(\mathbf{b}_{\alpha, 1}\right)^{\beta} \in \mathcal{B}_{\alpha / \beta, 1}$.

(5) Take $\mu \in \mathcal{B}_{\alpha, 1}$, then $\mu$ is of the form $\nu^{\otimes 1 / \alpha} \otimes \mathbf{b}_{\alpha, 1}$, so that

$$
\mu^{\bigotimes t}=\left(\nu^{\otimes 1 / \alpha}\right)^{\bigotimes t} \otimes\left(\mathbf{b}_{\alpha, 1}\right)^{\bigotimes t}=\nu^{\bigotimes t / \alpha} \otimes \mathbf{b}_{\frac{\alpha}{t(1-\alpha)+\alpha}, 1},
$$

where we used (3.15) on the last equality. We define $\lambda=\nu^{\otimes \frac{t}{t(1-\alpha)+\alpha}}$, to obtain $\mu^{\bigotimes t}=\lambda^{\bigotimes \frac{t(1-\alpha)+\alpha}{\alpha}} \bigotimes \mathbf{b}_{\frac{\alpha}{t(1-\alpha)+\alpha}, 1} \in \mathcal{B}_{\frac{\alpha}{t(1-\alpha)+\alpha}, 1}$. Note that $\frac{t}{t(1-\alpha)+\alpha}=\frac{t}{t-(t-1) \alpha} \geq 1$, and so $\lambda$ exists as a probability measure.

(6) This follows from the fact that $\left(\mathbf{b}_{\alpha, 1}\right)^{-1}=\mathbf{b}_{\alpha, 1}$ since $\mathbf{b}_{\alpha, 1}$ is the law of the quotient of two classical independent, identically distributed positive stable random variables (see (4.16)). 
We study the behavior of the probability density function at $x=0$.

Proposition 4.10. Let $(\alpha, \rho)$ be admissible. If $\tau=\mu \circledast \mathbf{b}_{\alpha, \rho} \in \mathcal{B}_{\alpha, \rho}$ and $\tau \neq \delta_{0}$, then the density function $p_{\mu, \alpha, \rho}(x)$ of absolutely continuous part of $\tau$ satisfies

$$
\liminf _{x \downarrow 0} \frac{p_{\mu, \alpha, \rho}(x)}{x^{\alpha-1}} \in(0, \infty], \quad \text { if } \rho \neq 0,0<\alpha \leq 1 \text { or } \rho \neq 1 / \alpha, 1<\alpha<2,
$$

$$
\liminf _{x \uparrow 0} \frac{p_{\mu, \alpha, \rho}(x)}{x^{\alpha-1}} \in(0, \infty], \quad \text { if } \rho \neq 1,0<\alpha \leq 1 \text { or } \rho \neq 1-1 / \alpha, 1<\alpha<2 .
$$

In particular, $\mathbf{b}_{\beta, \rho} \notin \mathcal{B}_{\alpha, \rho}$ if $0<\alpha<\beta \leq \min (1 / \rho, 1 /(1-\rho))$.

Proof. We can find an interval $[a, b]$ of $(0, \infty)$ such that $\mu([a, b])>0$. Let $\rho \neq 0, \alpha \leq$ 1. Then for $x>0$, we get

$$
\begin{aligned}
p_{\mu, \alpha, \rho}(x) & =(1-\mu(\{0\})) \frac{\sin (\pi \rho \alpha)}{\pi} \int_{(0, \infty)} \frac{x^{\alpha-1} t^{\alpha}}{x^{2 \alpha}+2(x t)^{\alpha} \cos (\pi \rho \alpha)+t^{2 \alpha}} \mu(d t) \\
& \geq(1-\mu(\{0\})) \frac{\sin (\pi \rho \alpha)}{\pi} \int_{[a, b]} \frac{x^{\alpha-1} t^{\alpha}}{x^{2 \alpha}+2(x t)^{\alpha}|\cos (\pi \rho \alpha)|+t^{2 \alpha}} \mu(d t) \\
& \geq(1-\mu(\{0\})) \frac{\sin (\pi \rho \alpha)}{\pi} \mu([a, b]) \frac{x^{\alpha-1} a^{\alpha}}{x^{2 \alpha}+2 x^{\alpha} b^{\alpha}|\cos (\pi \rho \alpha)|+b^{2 \alpha}},
\end{aligned}
$$

which leads to the conclusion (4.14). The other cases can be treated similarly.

If $0<\alpha<\beta$, then for $\mathbf{b}_{\beta, \rho}$ we have $\lim _{x \downarrow 0} \frac{p_{\beta, \rho}(x)}{x^{\alpha-1}}=0$. Hence $\mathbf{b}_{\beta, \rho} \notin \mathcal{B}_{\alpha, \rho}$.

Proposition 4.11. Let $\rho \in[0,1]$. Then $\mathcal{B}_{\alpha, \rho} \subset \mathcal{B}_{\beta, \rho}$ if $0<\alpha<\beta \leq \min (1 / \rho, 1 /(1-$ $\rho)$ ), where we understand that $1 / 0=\infty$. The inclusion is strict.

Proof. The relation $\mathbf{b}_{\alpha, \rho} \circledast\left(\mathbf{b}_{\beta, 1}\right)^{1 / \alpha}=\mathbf{b}_{\alpha \beta, \rho}$ in Proposition 4.8 implies this inclusion. The strictness of the inclusions follows from Proposition 4.10.

4.2. Connections between Classical, Free and Boolean Stable Laws. We want to point out some relations between Boolean, free and classical stable laws. As noted in the last paragraph of [2], there is an interplay among free, Boolean and classical stable laws. We have the identity $\mathbf{f}_{\alpha, 1} \otimes\left(\mathbf{f}_{\alpha, 1}\right)^{-1}=\mathbf{n}_{\alpha, 1} \circledast\left(\mathbf{n}_{\alpha, 1}\right)^{-1}$ for $\alpha \in(0,1]$ as proved in Proposition A4.4 of [15]. Moreover, this coincides with a Boolean stable law:

$$
\mathbf{b}_{\alpha, 1}=\mathbf{f}_{\alpha, 1} \otimes\left(\mathbf{f}_{\alpha, 1}\right)^{-1}=\mathbf{n}_{\alpha, 1} \circledast\left(\mathbf{n}_{\alpha, 1}\right)^{-1}, \quad \alpha \in(0,1] .
$$

This relation can be generalized as follows.

Proposition 4.12. The following formulas hold true.

$$
\begin{array}{ll}
\mathbf{b}_{\alpha, \rho}=\mathbf{f}_{\alpha, \rho} \otimes\left(\mathbf{f}_{\alpha, 1}\right)^{-1}, & \alpha \in(0,1], \rho \in\{0,1 / 2,1\}, \\
\mathbf{b}_{\alpha, \rho}=\mathbf{n}_{\alpha, \rho} \circledast\left(\mathbf{n}_{\alpha, 1}\right)^{-1}, & \alpha \in(0,1], \rho \in[0,1] .
\end{array}
$$

Remark 4.13. These relations do not hold for $\alpha>1$ since $\mathbf{n}_{\alpha, 1}$ and $\mathbf{f}_{\alpha, 1}$ are not defined.

Proof. (4.17) follows from (3.17) and the fact

$$
\left(\mathbf{f}_{\alpha, 1}\right)^{-1}=\boldsymbol{\pi}^{\otimes \frac{1-\alpha}{\alpha}}
$$


which can be proved from (2.6), (3.9) and (3.13).

(4.18) From Proposition 4.8, we have, on one hand, that

$$
\mathbf{b}_{\alpha, \rho}=\mathbf{b}_{\alpha, 1} \circledast \mathbf{c}_{\rho} .
$$

On the other hand, from [49, Theorem 3.3.1], we get

$$
\mathbf{n}_{\alpha, \rho}=\mathbf{n}_{\alpha, 1} \circledast \mathbf{c}_{\rho} .
$$

Hence we get (4.18) by multiplying (4.16) by $\mathbf{c}_{\rho}$.

Here we collect some identities and properties for $\mathbf{b}_{\alpha, \rho}, \mathbf{f}_{\alpha, \rho}, \mathbf{n}_{\alpha, \rho}$, including known results which may bring some insight into relationship between different kinds of stable law.

Theorem 4.14. The following relations hold.

$$
\begin{array}{ll}
\left(\mathbf{b}_{\beta, 1}\right)^{1 / \alpha} \circledast \mathbf{b}_{\alpha, \rho}=\mathbf{b}_{\alpha \beta, \rho}, & (\alpha, \rho) \in \mathfrak{A}, \beta \in(0,1], \\
\left(\mathbf{b}_{\beta, 1}\right)^{\bigotimes 1 / \alpha} \otimes \mathbf{b}_{\alpha, \rho}=\mathbf{b}_{\alpha \beta, \rho}, & (\alpha, \rho) \in \mathfrak{A}, \beta \in(0,1], \rho \in\{0,1 / 2,1\}, \\
\mathbf{b}_{\beta, 1} \circlearrowright \mathbf{b}_{\alpha, \rho}=\mathbf{b}_{\alpha \beta, \rho}, & (\alpha, \rho) \in \mathfrak{A}, \beta \in(0,1], \\
\left(\mathbf{n}_{\beta, 1}\right)^{1 / \alpha} \circledast \mathbf{n}_{\alpha, \rho}=\mathbf{n}_{\alpha \beta, \rho}, & (\alpha, \rho) \in \mathfrak{A}, \beta \in(0,1], \\
\left(\mathbf{f}_{\beta, 1}\right)^{\bigotimes 1 / \alpha} \otimes \mathbf{f}_{\alpha, \rho}=\mathbf{f}_{\alpha \beta, \rho}, & (\alpha, \rho) \in \mathfrak{A}, \beta \in(0,1], \rho \in\{0,1 / 2,1\} .
\end{array}
$$

Moreover, we have the following properties:

$$
\begin{aligned}
& \eta_{\mu^{1 / \alpha} \circledast \mathbf{b}_{\alpha, \rho}}(z)=\eta_{\mu}\left(-\left(e^{i \rho \pi} z\right)^{\alpha}\right), \quad z \in \mathbb{C}^{-},(\alpha, \rho) \in \mathfrak{A}, \\
& \text { (4.25) } \mathcal{M}_{\mu^{1 / \alpha} \circledast \mathbf{n}_{\alpha, \rho}}(z)=\mathcal{M}_{\mu}\left(-\left(e^{i \rho \pi} z\right)^{\alpha}\right), \quad z \in i(-\infty, 0),(\alpha, \rho) \in \mathfrak{A} \text {, } \\
& \mathcal{C}_{\mu^{\boxplus 1 / \alpha} \boxplus \mathbf{f}_{\alpha, \rho}}^{\boxplus}(z)=\mathcal{C}_{\mu}^{\boxplus}\left(-\left(e^{i \rho \pi} z\right)^{\alpha}\right), \quad z \in\left(\Gamma_{a, b}\right)^{-1}, \alpha \leq 1, \rho \in\{0,1 / 2,1\}
\end{aligned}
$$

for some $a, b>0$ depending on $\mu, \alpha, \rho$. In particular, the maps $\mathbf{B}_{\alpha, \rho}, \mathbf{N}_{\alpha, \rho}, \mathbf{F}_{\alpha, \rho}$ : $\mathcal{P}_{+} \rightarrow \mathcal{P}$ defined by

$$
\begin{array}{ll}
\mathbf{B}_{\alpha, \rho}(\mu)=\mu^{1 / \alpha} \circledast \mathbf{b}_{\alpha, \rho}, & (\alpha, \rho) \in \mathfrak{A}, \\
\mathbf{N}_{\alpha, \rho}(\mu)=\mu^{1 / \alpha} \circledast \mathbf{n}_{\alpha, \rho}, & (\alpha, \rho) \in \mathfrak{A}, \\
\mathbf{F}_{\alpha, \rho}(\mu)=\mu^{\boxplus 1 / \alpha} \boxplus \mathbf{f}_{\alpha, \rho}, & \alpha \in(0,1], \rho \in\{0,1 / 2,1\}
\end{array}
$$

are homomorphisms with respect to $\uplus, *, \boxplus$, respectively.

Remark 4.15. We can understand that for $\alpha \leq 1$, the formulas (4.19) and (4.20) are consequences of the formulas (4.22) and (4.23) respectively, together with the identities in Proposition 4.12. This argument is not available for $\alpha>1$ since Proposition 4.12 is no longer true.

Proof. (4.19) was proved in Proposition 4.8. (4.20) and (4.21) follow from (4.19), (4.9) and (4.10). (4.22) is known; see [49, Theorem 3.3.1]. (4.23) is proved by the direct computation of the $S$-transform (3.9).

The formula (4.24) is exactly (4.5). For the formula (4.25), let $X, N_{\alpha, \rho}$ be independent random variables following the laws $\mu, \mathbf{n}_{\alpha, \rho}$ respectively. By using (2.12), we have the formula

$$
\begin{aligned}
\mathcal{M}_{\mu^{1 / \alpha} \circledast \mathbf{n}_{\alpha, \rho}}(z) & =\mathbb{E}\left[e^{\left.z X^{1 / \alpha} N_{\alpha, \rho}\right]}=\mathbb{E}\left[\exp \left(-e^{i \rho \alpha \pi}\left(z X^{1 / \alpha}\right)^{\alpha}\right)\right]\right. \\
& =\mathbb{E}\left[\exp \left(-X\left(e^{i \rho \pi} z\right)^{\alpha}\right)\right]=\mathcal{M}_{\mu}\left(-\left(e^{i \rho \pi} z\right)^{\alpha}\right), \quad z \in i(-\infty, 0) .
\end{aligned}
$$


For the formula (4.26), we compute

$$
S_{\mu^{\boxplus 1 / \alpha} \otimes \mathbf{f}_{\alpha, \rho}}(z)=-e^{-i \rho \pi}(-z)^{\frac{1-\alpha}{\alpha}} S_{\mu}(z)^{1 / \alpha},
$$

and hence

$$
z S_{\mu^{\bigotimes 1 / \alpha} \otimes \mathbf{f}_{\alpha, \rho}}(z)=e^{-i \rho \pi}\left(-z S_{\mu}(z)\right)^{1 / \alpha} .
$$

Due to [6, 35], the relation $\mathcal{C}_{\nu}^{\boxplus}\left(z S_{\nu}(z)\right)=z$ holds for $\nu \in \mathcal{P}_{+}$or $\nu \in \mathcal{P}_{s}$ in an open neighborhood $U$ of $(-a, 0)$ for some $a>0$. Therefore $f(z)=z S_{\nu}(z)$ is univalent in $U$ and $\mathcal{C}_{\nu}^{\boxplus}$ is univalent in $f(U)$ which contains an interval $(-b, 0)$ if $\nu \in \mathcal{P}_{+}$and an interval $i(0, c)$ if $\nu \in \mathcal{P}_{s}$. Hence we have $z S_{\nu}(z)=\left(\mathcal{C}_{\nu}^{\boxplus}\right)^{-1}(z)$ and then

$$
\left(\mathcal{C}_{\mu^{\boxplus 1 / \alpha} \boxplus \mathbf{f}_{\alpha, \rho}}^{\boxplus}\right)^{-1}(z)=e^{-i \rho \pi}\left(-\left(\mathcal{C}_{\mu}^{\boxplus}\right)^{-1}(z)\right)^{1 / \alpha}, \quad z \in(-s, 0)
$$

for some $s>0$. The formula (4.26) follows after some computation and by analytic continuation.

As a final comment regarding multiplicative properties of stable laws, we want to point out that the formulas (4.20) and (4.23) are relatives of the reproducing properties

$$
\begin{aligned}
& \mathbf{f}_{1 /(1+t), 1} \otimes \mathbf{f}_{1 /(1+s), \rho}=\mathbf{f}_{1 /(1+s+t), \rho}, \\
& \mathbf{b}_{1 /(1+t), 1} \otimes \mathbf{b}_{1 /(1+s), \rho}=\mathbf{b}_{1 /(1+s+t), \rho}
\end{aligned}
$$

for $s, t \geq 0,(\alpha, \rho) \in \mathfrak{A}, \rho \in\{0,1 / 2,1\}$. The formula (4.27) was established in [15] for $\rho=1$ and in [6] for $\rho=1 / 2$, and the formula (4.28) was established in [2]. We expect these formulas, as well as (3.17), (4.9), (4.17), (4.20) and (4.23), to be true for general $\rho$, but the $S$-transform is not yet available in the general case.

4.3. Classical and Multiplicative Infinite Divisibility. We prove the ID part of Theorem 1.1 and the following paragraph. Before proving it, let us recall some facts about exponential mixtures. See [43] for further details.

Definition 4.16. A measure is said to be an exponential mixture if $\mu$ is distributed as the random variable $X E$, where $E$ follows the exponential distribution with density $e^{-x} 1_{(0, \infty)}(x)$ and $X$ is any random variable independent of $X$. If $X$ is positive then $\mu$ is called a positive exponential mixture. We denote by $\mathcal{E} \mathcal{M}$ the set of exponential mixtures.

Some properties of exponential functions are the following.

(1) A positive random variable $X$ is an exponential mixture if and only if $X$ has a completely monotone density.

(2) If $X$ is a positive exponential mixture then $X^{\alpha}$ is also for $\alpha \geq 1$.

(3) If $X \in \mathcal{E} \mathcal{M}$ and $Y$ is independent of $X$, then $X Y \in \mathcal{E M}$.

The importance of exponential mixtures in this paper comes from the following theorem.

Theorem 4.17. $\mathcal{E} \mathcal{M} \subset \mathcal{I D}(*)$.

Now we are ready to prove part of Theorem 1.1 .

Theorem 4.18. (1) If $\alpha \in(0,1 / 2], \rho \in[0,1]$, then $\mathcal{B}_{\alpha, \rho} \subset \mathcal{E} \mathcal{M}$.

(2) If $\alpha \in(0,1], \rho=1 / 2$, then $\mathcal{B}_{\alpha, \rho} \subset \mathcal{I D}(*)$.

(3) If $\rho \neq 1 / 2$, then $\mathcal{B}_{1, \rho} \not \subset \mathcal{I D}(*)$. 
Proof. (1) Clearly it is enough to show that $\mathbf{b}_{\alpha, \rho}$ itself is an exponential mixture for $\alpha \leq 1 / 2$. It is proved in [2] that $\mathbf{b}_{\alpha, 1}$ is a positive exponential mixture. We now use the identity in Proposition 4.8: $\mathbf{b}_{\beta, \rho} \circledast\left(\mathbf{b}_{1 / 2,1}\right)^{1 / \beta}=\mathbf{b}_{\beta / 2, \rho}$. Since $\left(\mathbf{b}_{1 / 2,1}\right)^{1 / \beta}$ is a positive exponential mixture for $\beta \leq 1$, we see that $\mathbf{b}_{\alpha, \rho}$ is an exponential mixture for $\alpha \leq 1 / 2$.

(2) Any mixture of a symmetric Cauchy distribution is ID from Theorem IV.10.5 in Steutel and van Harn [43]. From Remark 4.2 and Proposition 4.8 we have $\mathbf{b}_{\alpha, 1 / 2}=\mathbf{b}_{\alpha, 1} \circledast \mathbf{c}_{1 / 2}$ and hence $\mu \circledast \mathbf{b}_{\alpha, 1 / 2}$ is also a mixture of the symmetric Cauchy distribution $\mathbf{c}_{1 / 2}$.

(3) Let $p \in(0,1), \rho \neq 1 / 2$ and consider the law $\left(p \delta_{0}+(1-p) \delta_{1}\right) * \mathbf{b}_{1, \rho}$. Its Fourier transform can be computed as

$$
\mathcal{F}_{\left(p \delta_{0}+(1-p) \delta_{1}\right) * \mathbf{b}_{1, \rho}}(z)=p+(1-p) e^{-(\sin \rho \pi)|z|+i(\cos \rho \pi) z}, z \in \mathbb{R},
$$

and in particular

$$
\mathcal{F}_{\left(p \delta_{0}+(1-p) \delta_{1}\right) * \mathbf{b}_{1, \rho}}\left(\frac{\pi}{\cos \rho \pi}\right)=p-(1-p) e^{-\pi \tan \rho \pi} .
$$

If we take $p:=\frac{1}{1+e^{\pi \tan \rho \pi}}$, then $\mathcal{F}_{\mathbf{b}_{1, \rho} *\left(p \delta_{0}+(1-p) \delta_{1}\right)}\left(\frac{\pi}{\cos \rho \pi}\right)=0$, which implies that $\left(p \delta_{0}+(1-p) \delta_{1}\right) * \mathbf{b}_{1, \rho} \notin \mathcal{I D}(*)$ from Proposition IV.2.4 in [43].

Problem 4.19. Determine the possible pairs $(\alpha, \rho)$ completely so that $\mathcal{B}_{\alpha, \rho} \subset$ $\mathcal{I D}(*)$.

Now, we prove infinite divisibility of Boolean stable laws in the multiplicative case.

Proposition 4.20 (Multiplicative infinite divisibility). The positive Boolean stable law $\mathbf{b}_{\alpha, 1}$ is infinitely divisible with respect to the convolutions $\circledast, \bigotimes, \circlearrowright$ for any $\alpha \leq 1$.

Proof. The stable distribution $\mathbf{n}_{\alpha, 1}$ is $\circledast$-infinitely divisible (see [49, Theorem 3.5.1]), and hence so is $\left(\mathbf{n}_{\alpha, 1}\right)^{-1}$. Therefore $\mathbf{b}_{\alpha, 1}=\left(\mathbf{n}_{\alpha, 1}\right)^{-1} \circledast \mathbf{n}_{\alpha, 1}$ is $\circledast$-infinitely divisible too (see (4.16)). The $\bigotimes$-infinite divisibility follows from (3.15) and the $\circlearrowright$-infinite divisibility follows from (3.16).

4.4. Free Infinite Divisibility of $\mathcal{B}_{\alpha, \rho}$. We prove the free part of Theorem 1.1 and the following paragraph. We start from short proofs of the free infinite divisibility of $\mathcal{B}_{\alpha, 1}$ and $\mathcal{B}_{\alpha, 1 / 2}$ by using Proposition 3.7.

Proposition 4.21. (1) For $\alpha \leq 1 / 2, \rho \in\{0,1 / 2,1\}$ and $\mu \in \mathcal{P}_{+}$, the measure $\mu^{1 / \alpha} \circledast \mathbf{b}_{\alpha, \rho}$ is a compound free Poisson with rate 1 and jump distribution $\mu^{\bigotimes 1 / \alpha} \otimes \pi^{\bigotimes \frac{1-2 \alpha}{\alpha}} \otimes \mathbf{f}_{\alpha, \rho}$, and hence $\mu^{1 / \alpha} \circledast \mathbf{b}_{\alpha, \rho}$ is FID.

(2) For $\alpha \leq 2 / 3$ and $\mu \in \mathcal{P}_{+}$, the probability measure $\mu^{1 / \alpha} \circledast \mathbf{b}_{\alpha, 1 / 2}$ is a compound free Poisson with rate 1 and jump distribution $\mu^{\bigotimes 1 / \alpha} \nabla \pi^{\bigotimes \frac{2-3 \alpha}{2 \alpha}} \nabla \operatorname{Sym}\left(\sqrt{\mathbf{f}_{\alpha / 2,1}}\right)$, and hence it is FID.

Proof. These are obvious from (4.9), (3.17), (3.18) and Remark 2.2.

The complete determination of the free infinite divisibility of $\mathcal{B}_{\alpha, \rho}$ requires the ideas of [2] and [18].

Definition 4.22. A probability measure $\mu$ is said to be in class $\mathcal{U I}$ if $F_{\mu}^{-1}$, defined in a domain $\Gamma_{\alpha, \beta}$, has an analytic continuation which is univalent in $\mathbb{C}^{+}$. From the 
Riemann mapping theorem, $\mu \in \mathcal{U} \mathcal{I}$ if and only if there exists a domain $\mathbb{C}^{+} \subset D \subset$ $\mathbb{C}$ such that $F_{\mu}$ extends to an analytic bijection $\tilde{F}_{\mu}$ from $D$ onto $\mathbb{C}^{+}$.

The importance of this class is given by the following lemma (implicitly used in $[12])$.

Lemma 4.23. [3] If $\mu \in \mathcal{U I}$ then $\mu$ is FID.

The following result was shown in [18, Proposition 2.1].

Lemma 4.24. A probability measure $\mu$ on $\mathbb{R}$ is in $\mathcal{U} \mathcal{I}$ if there exists a simple, continuous curve $\gamma=(\gamma(t))_{t \in \mathbb{R}} \subset \mathbb{C}^{-} \cup \mathbb{R}$ with the following properties:

(A) $\lim _{t \rightarrow \infty}|\gamma(t)|=\lim _{t \rightarrow-\infty}|\gamma(t)|=\infty$;

(B) $F_{\mu}$ extends to an analytic function $\tilde{F}_{\mu}$ in $D(\gamma)$ which is continuous on $\overline{D(\gamma)}$, where $D(\gamma)$ denotes the simply connected open set containing $\mathbb{C}^{+}$with boundary $\gamma$

(C) $\tilde{F}_{\mu}(\gamma) \subset \mathbb{C}^{-} \cup \mathbb{R}$;

(D) $\tilde{F}_{\mu}(z)=z+o(z)$ uniformly as $z \rightarrow \infty, z \in D(\gamma)$.

The following result completes the free part of Theorem 1.1.

Theorem 4.25. The following statements hold.

(1) If $\alpha \in(0,1 / 2]$ and $\rho \in[0,1]$, then $\mathcal{B}_{\alpha, \rho} \subset \mathcal{U I} \subset \mathcal{I D}(\boxplus)$.

(2) If $\alpha \in(1 / 2,2 / 3]$ and $\rho \in[2-1 / \alpha, 1 / \alpha-1]$, then $\mathcal{B}_{\alpha, \rho} \subset \mathcal{I D}(\boxplus)$.

(3) Otherwise, $\mathcal{B}_{\alpha, \rho} \not \subset \mathcal{I D}(\boxplus)$.

Moreover, if $(\alpha, \rho)$ satisfies the assumptions of (1) or (2), then any probability measure $\nu \in \mathcal{B}_{\alpha, \rho}$ has free divisibility indicator infinity.

Proof. Let $B_{\alpha, \rho}$ and $X$ be classically independent random variables following $\mathbf{b}_{\alpha, \rho}$ and a probability measure $\mu \in \mathcal{P}_{+}$, respectively. We may assume that $X$ is discrete and takes only a finitely many number of positive values, so in particular $a \leq X \leq b$ for some $0<a<b$. The general case follows from approximation since the set $\mathcal{U I}$ (resp. $\mathcal{I D}(\boxplus)$ ) is closed with respect to the weak convergence [3] (resp. see e.g. [10, Theorem 3.8] and [39, Lemma 7.8]).

Using (4.6), we have

$$
G_{X B_{\alpha, \rho}}(z)=\mathbb{E}\left[\frac{1}{z+e^{i \alpha \rho \pi} X^{\alpha} z^{1-\alpha}}\right], \quad z \in \mathbb{C}^{+} .
$$

We define

$$
\begin{aligned}
& \theta_{\alpha, \rho}:=-\frac{\alpha \rho \pi}{1-\alpha}, \phi_{\alpha, \rho}:=\frac{(1-\rho \alpha) \pi}{1-\alpha} \\
& \ell_{\theta}:=\left\{r e^{i \theta}: r>0\right\}, \quad \theta \in \mathbb{R}, \\
& \gamma:=\ell_{\theta_{\alpha, \rho}} \cup\{0\} \cup \ell_{\phi_{\alpha, \rho}} .
\end{aligned}
$$

(1) Assume moreover that $\alpha \in(0,1 / 2)$; the case $\alpha=1 / 2$ follows by approximation. It then holds that $\theta_{\alpha, \rho} \in(-\pi, 0], \phi_{\alpha, \rho} \in[\pi, 2 \pi)$ and $\phi_{\alpha, \rho}-\theta_{\alpha, \rho} \in(\pi, 2 \pi)$. Note that $D(\gamma)=\mathbb{C}_{\left(\theta_{\alpha, \rho}, \phi_{\alpha, \rho}\right)}$.

We will show that the curve $\gamma$ satisfies the assumptions in Lemma 4.24. Condition (A) is clear. For condition (B), we first show that

(*) $G_{X B_{\alpha, \rho}}$ extends analytically to a function $\tilde{G}$ in $D(\gamma)$ which is continuous on $\overline{D(\gamma)} \backslash\{0\}$, and also $\tilde{G}$ does not have a zero in $\overline{D(\gamma)} \backslash\{0\}$. 
In view of (4.29), it suffices to show that for any $x>0$ and $z \in \overline{D(\gamma)} \backslash\{0\}$, the point $w(x, z):=z+x^{\alpha} e^{i \alpha \rho \pi}(z)_{\left(\theta_{\alpha, \rho}, \phi_{\alpha, \rho}\right)}^{1-\alpha}$ is not zero. Indeed, when $z \in \mathbb{C}^{+}, w(x, z)$ is not zero since $w(x, z)=F_{x B_{\alpha, \rho}}(z) \in \mathbb{C}^{+}$. When $z=r e^{i \theta}, \theta \in\left[\theta_{\alpha, \rho}, 0\right]$, we compute the difference of the arguments of the points $z, x^{\alpha} e^{i \alpha \rho \pi}(z)_{\left(\theta_{\alpha, \rho}, \phi_{\alpha, \rho}\right)}^{1-\alpha}$ :

$$
0<(1-\alpha) \theta+\alpha \rho \pi-\theta \leq \frac{\alpha}{1-\alpha} \rho \pi<\pi
$$

This shows, for each $z=r e^{i \theta}, \theta \in\left[\theta_{\alpha, \rho}, 0\right]$, there exists a line $L_{z}$ passing 0 such that for any $x>0$ the points $z, x^{\alpha} e^{i \alpha \rho \pi}(z)_{\left(\theta_{\alpha, \rho}, \phi_{\alpha, \rho}\right)}^{1-\alpha}$ lie in the same open half-plane $H_{z}$ with boundary $L_{z}$, and hence $w(x, z)$ lies in $H_{z}$ too, so $w(x, z) \neq 0$. When $z=r e^{i \theta}, \theta \in\left[\pi, \phi_{\alpha, \rho}\right]$ we get similarly

$$
0>(1-\alpha) \theta+\alpha \rho \pi-\theta \geq-\frac{\alpha}{1-\alpha}(1-\rho) \pi>-\pi .
$$

From a similar reasoning, $w(x, z) \neq 0$. Since $w(x, z)$ is continuous with respect to $x$, we get $\inf _{x \in[a, b]}|w(x, z)|>0$. Hence we can define the analytic continuation of $G_{X B_{\alpha, \rho}}$ by

$$
\tilde{G}(z)=\mathbb{E}\left[\frac{1}{z+e^{i \alpha \rho \pi} X^{\alpha}(z)_{\left(\theta_{\alpha, \rho}, \phi_{\alpha, \rho}\right)}^{1-\alpha}}\right], \quad z \in D(\gamma) .
$$

This extends continuously to $\overline{D(\gamma)} \backslash\{0\}$. The arguments around (4.30) and (4.31) actually show that $\tilde{G}(z) \neq 0$ for $z \in \overline{D(\gamma)} \backslash\{0\}$, because $1 / w(x, z)$ lies in the halfplane $\left(H_{z}\right)^{-1}$ for any $x>0$ and so $\tilde{G}(z)=\mathbb{E}[1 / w(X, z)] \in\left(H_{z}\right)^{-1}$ too. Thus we established $(*)$.

Let $\tilde{F}(z):=1 / \tilde{G}(z)$. Then $\tilde{F}$ is analytic in $D(\gamma)$ and continuous on $\overline{D(\gamma)} \backslash\{0\}$ from $(*)$. Moreover, since $X$ takes only finitely many values, it is easy to see that $\lim _{z \rightarrow 0, z \in D(\gamma)} \tilde{G}(z)=\infty$, and hence $\tilde{F}$ extends to a continuous function on $\overline{D(\gamma)}$. This is condition (B).

For condition (C), take $r>0$ and then

$$
\begin{aligned}
& \frac{1}{r e^{i \theta_{\alpha, \rho}}+e^{i \alpha \rho \pi} X^{\alpha}\left(r e^{i \theta_{\alpha, \rho}}\right)_{\left(\theta_{\alpha, \rho}, \phi_{\alpha, \rho}\right)}^{1-\alpha}}=\frac{1}{r e^{i \theta_{\alpha, \rho}}+X^{\alpha} r^{1-\alpha}} \in \mathbb{C}^{+} \cup(0, \infty), \\
& \frac{1}{r e^{i \phi_{\alpha, \rho}}+e^{i \alpha \rho \pi} X^{\alpha}\left(r e^{i \phi_{\alpha, \rho}}\right)_{\left(\theta_{\alpha, \rho}, \phi_{\alpha, \rho}\right)}^{1-\alpha}}=\frac{1}{r e^{i \phi_{\alpha, \rho}}-X^{\alpha} r^{1-\alpha}} \in \mathbb{C}^{+} \cup(-\infty, 0) .
\end{aligned}
$$

We take the expectation and use (4.29) to obtain $\tilde{G}(\gamma \backslash\{0\}) \subset \mathbb{C}^{+} \cup \mathbb{R} \backslash\{0\}$. Recall that $\tilde{F}(0)=0$ and so we have condition $(\mathrm{C})$.

Finally, since $X$ is bounded, it is easy to show that

$$
z\left(\tilde{G}(z)-\frac{1}{z}\right)=-\mathbb{E}\left[\frac{e^{i \alpha \rho \pi} X^{\alpha}(z)_{\left(\theta_{\alpha, \rho}, \phi_{\alpha, \rho}\right)}^{-\alpha}}{1+e^{i \alpha \rho \pi} X^{\alpha}(z)_{\left(\theta_{\alpha, \rho}, \phi_{\alpha, \rho}\right)}^{-\alpha}}\right]=o(1)
$$

uniformly as $z \rightarrow \infty, z \in D(\gamma)$. This shows condition (D). From Lemma 4.24, the law of $X B_{\alpha, \rho}$ is in $\mathcal{U} \mathcal{I}$.

(2) Assume moreover that $\rho \in(2-1 / \alpha, 1 / \alpha-1)$. Note now that $\theta_{\alpha, \pi} \in$ $(-\pi, 0), \phi_{\alpha, \rho} \in(\pi, 2 \pi)$. Since now $\phi_{\alpha, \rho}-\theta_{\alpha, \rho}>2 \pi$, the sector $\mathbb{C}_{\left(\theta_{\alpha, \rho}, \phi_{\alpha, \rho}\right)}$ coincides with $\mathbb{C} \backslash\{0\}$ as a subset of $\mathbb{C}$, we have to modify Lemma 4.24. We use the Riemannian surface corresponding to the interval $\left(\theta_{\alpha, \rho}, \phi_{\alpha, \rho}\right)$ of arguments and divide the domain into three parts: $\mathbb{C}_{\left(\theta_{\alpha, \rho}, \rho \pi\right)}, \mathbb{C}_{\left(\rho \pi, \phi_{\alpha, \rho}\right)}$ and an open neighborhood of $\ell_{\rho \pi}$. 
We denote by $\tilde{G}_{1}, \tilde{G}_{2}, \tilde{G}_{3}$ analytic maps in these three domains respectively such that each coincides with $G_{X B_{\alpha, \rho}}$ in the intersection of each domain and $\mathbb{C}^{+}$, and we denote by $\tilde{F}_{i}$ their reciprocals. Note that we can define the analytic continuations $\tilde{G}_{1}, \tilde{G}_{2}$ along the same line of the previous case $(1)$; the inequalities $(4.30),(4.31)$ are still true thanks to the assumption $\rho \in(2-1 / \alpha, 1 / \alpha-1)$, and so the functions $w_{1}(x, z):=z+x^{\alpha} e^{i \alpha \rho \pi}(z)_{\left(\theta_{\alpha, \rho}, \rho \pi\right)}^{1-\alpha}$ and $w_{2}(x, z):=z+x^{\alpha} e^{i \alpha \rho \pi}(z)_{\left(\rho \pi, \phi_{\alpha, \rho}\right)}^{1-\alpha}$ do not vanish. Hence we have the expression for $\tilde{G}_{1}$ as

$$
\tilde{G}_{1}(z)=\mathbb{E}\left[\frac{1}{z+e^{i \alpha \rho \pi} X^{\alpha}(z)_{\left(\theta_{\alpha, \rho}, \rho \pi\right)}^{1-\alpha}}\right]
$$

and similarly for $\tilde{G}_{2}$. The map $\tilde{G}_{3}$ is just the restriction of $G_{X B_{\alpha, \rho}}$. Note that $\tilde{F}_{3}\left(\ell_{\rho \pi}\right)=\ell_{\rho \pi}$ and $\tilde{F}_{3}$ is univalent in an open neighborhood $D_{3}$ of $\ell_{\rho \pi}$ from a direct computation of derivative of $\tilde{G}_{3}$. So the left compositional inverse $\left(\left.\tilde{F}_{3}\right|_{D_{3}}\right)^{-1}$ exists in an open neighborhood of $\ell_{\rho \pi}$.

We want to define a univalent inverse of $\tilde{F}_{1}$ in $\mathbb{C}_{(0, \rho \pi)}$. Now we take the curve $\gamma_{1}=\ell_{\theta_{\alpha, \rho}} \cup\{0\} \cup \ell_{\rho \pi}$ as the curve $\gamma$ in Lemma 4.24. We can check the conditions in Lemma 4.24 similarly to (1) except that we understand that $D\left(\gamma_{1}\right)=\mathbb{C}_{\left(\theta_{\alpha, \rho}, \rho \pi\right)}$ and we replace condition $(\mathrm{C})$ by $\tilde{F}_{1}\left(\gamma_{1}\right) \subset\left(\mathbb{C}_{(0, \rho \pi)}\right)^{c}$. Accordingly to these modifications, the conclusion of the lemma changes to: there is a domain $D_{1} \subset \mathbb{C}_{\left(\theta_{\alpha, \rho}, \rho \pi\right)}$ such that $\tilde{F}_{1}$ is a bijection from $D_{1}$ onto $\mathbb{C}_{(0, \rho \pi)}$. The proof of this fact is almost the same as [18, Proposition 2.1]. Hence its inverse map $\left(\left.\tilde{F}_{1}\right|_{D_{1}}\right)^{-1}$ exists in $\mathbb{C}_{(0, \rho \pi)}$. Similarly, the inverse $\left(\left.\tilde{F}_{2}\right|_{D_{2}}\right)^{-1}$ exists in $\mathbb{C}_{(\rho \pi, \pi)}$ for some $D_{2}$. Finally we define an analytic map $\tilde{F}^{-1}$ in $\mathbb{C}^{+}$by

$$
\tilde{F}^{-1}(z)= \begin{cases}\left(\left.\tilde{F}_{1}\right|_{D_{1}}\right)^{-1}(z), & z \in \mathbb{C}_{(0, \rho \pi)}, \\ \left(\left.\tilde{F}_{3}\right|_{D_{3}}\right)^{-1}(z), & z \in \ell_{\rho \pi} \\ \left(\left.\tilde{F}_{2}\right|_{D_{2}}\right)^{-1}(z), & z \in \mathbb{C}_{(\rho \pi, \pi)} .\end{cases}
$$

This map is not necessarily univalent, but we can show that $\tilde{\phi}(z):=\tilde{F}^{-1}(z)-z$ for $z \in \mathbb{C}^{+}$takes values in $\mathbb{C}^{-} \cup \mathbb{R}$; see the arguments in [23, Lemma 2.7] or in $[2$, Proposition 3.6]. Since $\tilde{\phi}$ is the analytic continuation of the Voiculescu transform $\phi_{X B_{\alpha, \rho}}$, the law of $X B_{\alpha, \rho}$ is FID from Theorem 2.1.

(3) As proved in [2], $\mathbf{b}_{\alpha, \rho} \notin \mathcal{I D}(\boxplus)$ in the following cases: $\alpha>1 ; \alpha \in(1 / 2,1)$ and $\rho \in\left[0, \frac{2 \alpha-1}{\alpha}\right) \cup\left(\frac{1-\alpha}{\alpha}, 1\right]$. The remaining case is $\alpha=1$ when $\mathbf{b}_{\alpha, \rho}$ is a Cauchy distribution, which itself is FID. However we can show $\mathcal{B}_{1, \rho} \not \subset \mathcal{I D}(\boxplus)$; see Proposition 5.8 .

For the final statement, take $\nu \in \mathcal{B}_{\alpha, \rho}$, which may be written as $\nu=\mu^{1 / \alpha} \circledast \mathbf{b}_{\alpha, \rho}$. For any $t>0$, from (4.5) we have

$$
\nu^{\uplus t}=\left(\mu^{\uplus t}\right)^{1 / \alpha} \circledast \mathbf{b}_{\alpha, \rho} \in \mathcal{B}_{\alpha, \rho} \subset \mathcal{I D}(\boxplus),
$$

and hence $\phi(\nu)=\infty$ from (2.11).

Remark 4.26. In the context of complex analysis, the map $F_{\mu \circledast \mathbf{b}_{\alpha, \rho}}$ may be useful because it has the invariant half line $\ell_{\rho \pi}=\left\{r e^{i \rho \pi}: r>0\right\}$. 
For a nonnegative finite measure $\sigma$ on $(0,1 / 2]$, the continuous Boolean convolution [2] is the probability measure $\mathbf{b}(\sigma)$ defined by

$$
\eta_{\mathbf{b}(\sigma)}(z)=-\int_{(0,1 / 2]}(-z)^{\alpha} \sigma(d \alpha)
$$

We can similarly prove the free infinite divisibility for the scale mixture $\mu \circledast \mathbf{b}(\sigma)$. However it turns out that $\mathbf{b}(\sigma)$ belongs to $\mathcal{B}_{1 / 2,1}$ as we see in Proposition 5.6.

\section{ExAmples}

5.1. Explicit Densities of Probability Measures in $\mathcal{B}_{1 / 2,1}$. The probability density function (4.1) of $\mu \circledast \mathbf{b}_{1 / 2,1}$ is in particular simply written as

$$
\frac{x^{-1 / 2}}{\pi} \int_{0}^{\infty} \frac{\sqrt{y}}{x+y} \mu(d y), \quad x>0 .
$$

By introducing the measure $\tau(d y)=\sqrt{y} \mu(d y)$, the density has the expression

$$
-\frac{x^{-1 / 2}}{\pi} G_{\tau}(-x), \quad x>0
$$

We will find explicit probability densities of this form.

Proposition 5.1. Let $\alpha \in(1 / 2,1]$ and $\alpha \beta \in(0,1]$. The generalized beta distribution of the second kind with density function

$$
c_{\alpha, \beta} \cdot \frac{x^{\alpha-3 / 2}}{\left(x^{\alpha \beta}+1\right)^{1 / \beta}} 1_{(0, \infty)}(x)
$$

belongs to the class $\mathcal{B}_{1 / 2,1}$, and hence it is in $\mathcal{I D}(\boxplus) \cap \mathcal{E M}$ from Theorems 4.18 and 4.25. Note that $c_{\alpha, \beta}>0$ is a normalizing constant.

Proof. From (5.1) and (5.2), it suffices to find a measure $\tau$ such that $y^{-1 / 2} \tau(d y)$ is a finite measure and that $-G_{\tau}(-x)=\frac{x^{\alpha-1}}{\left(x^{\alpha \beta}+1\right)^{1 / \beta}}$; then we may define $\mu=$ $c \cdot y^{-1 / 2} \tau(d y)$ for a normalizing constant $c>0$.

We define an analytic map

$$
G(z):=-\frac{(-z)^{\alpha-1}}{\left((-z)^{\alpha \beta}+1\right)^{1 / \beta}}, \quad z \in \mathbb{C} \backslash \mathbb{R}_{+}
$$

and we show that this is the Cauchy transform of a probability measure. Since $z G(z)=o(1)$ uniformly as $z \rightarrow \infty, z \in \mathbb{C}^{+}$, it suffices to show that $G$ maps $\mathbb{C}^{+}$into $\mathbb{C}^{-}$. For $z=r e^{i \theta}, r>0, \theta \in(0, \pi)$, we have

$\operatorname{Im}\left(G\left(r e^{i \theta}\right)\right)=-\operatorname{Im}\left(\frac{\left(r e^{i(\theta-\pi)}\right)^{\alpha-1}}{\left(\left(r e^{i(\theta-\pi)}\right)^{\alpha \beta}+1\right)^{1 / \beta}}\right)=-r^{\alpha-1} \operatorname{Im}\left(\frac{e^{i(1-\alpha)(\pi-\theta)}}{\left(r^{\alpha \beta} e^{-i \alpha \beta(\pi-\theta)}+1\right)^{1 / \beta}}\right)$.

Let $\varphi\left(r e^{i \theta}\right):=\arg \left(r^{\alpha \beta} e^{-i \alpha \beta(\pi-\theta)}+1\right)$ and $R\left(r e^{i \theta}\right):=\left|r^{\alpha \beta} e^{-i \alpha \beta(\pi-\theta)}+1\right|$. Since $\alpha \beta \in(0,1]$, it is easy to see that $r^{\alpha \beta} e^{-i \alpha \beta(\pi-\theta)}+1 \in \mathbb{C}^{-}$and $\varphi\left(r e^{i \theta}\right) \in(-\alpha \beta(\pi-$ $\theta), 0)$. We have the expression

$$
\operatorname{Im}\left(G\left(r e^{i \theta}\right)\right)=-r^{\alpha-1} R\left(r e^{i \theta}\right)^{-1 / \beta} \sin \left((1-\alpha)(\pi-\theta)-\varphi\left(r e^{i \theta}\right) / \beta\right) .
$$

Since $\varphi\left(r e^{i \theta}\right) \in(-\alpha \beta(\pi-\theta), 0)$, we get $0<(1-\alpha)(\pi-\theta)-\varphi\left(r e^{i \theta}\right) / \beta<\pi-\theta<\pi$, and hence $\operatorname{Im}\left(G\left(r e^{i \theta}\right)\right)<0$.

Now we know that there exists $\tau \in \mathcal{P}$ such that $G=G_{\tau}$. Since $G$ takes real values on $(-\infty, 0)$, it follows from the Stieltjes inversion that $\tau \in \mathcal{P}_{+}$. Both $\varphi$ and 
$R$ extend continuously to $\mathbb{C}^{+} \cup \mathbb{R} \backslash\{1\}\left(R\right.$ extends to $\mathbb{C}^{+} \cup \mathbb{R} . \varphi$ also extends to $\mathbb{C}^{+} \cup \mathbb{R}$ if $\alpha \beta<1$ ). Therefore (5.4) gives us

(5.5) $\lim _{y \downarrow 0} \operatorname{Im}(G(x+i y))=-x^{\alpha-1} R(x)^{-1 / \beta} \sin (\alpha \pi+\varphi(x) / \beta), \quad x>0, x \neq 1$.

By the Stieltjes inversion, $\tau$ has a density which behaves as $\frac{1+o(1)}{\pi} \sin (\alpha \pi) x^{\alpha-1}$ as $x \downarrow 0$, and hence $x^{-1 / 2} \tau(d x)$ is a finite measure for $\alpha \in(1 / 2,1]$. Thus $\mu \circledast \mathbf{b}_{1 / 2,1}$ has the density (5.3) thanks to the arguments in the first paragraph of the proof.

Proposition 5.2. Let $-1<a<1 / 2$. The probability measure with density

$$
c_{a} \cdot \frac{(1+x)^{a}-1}{a x^{3 / 2}} 1_{(0, \infty)}(x)
$$

belongs to $\mathcal{B}_{1 / 2,1}$, where $c_{a}>0$ is a normalizing constant. If $a=0$, this measure is understood as

$$
c_{0} \cdot \frac{\log (1+x)}{x^{3 / 2}} 1_{(0, \infty)}(x) d x .
$$

Proof. First consider $-1<a<0$ and let $\tau_{a}$ be the shifted beta distribution of the second kind with density $\frac{1}{B(1+a,-a)} \cdot \frac{(t-1)^{a}}{t}$ on $(1, \infty)$. From Example 3.3(4) in [23], we get $-G_{\tau_{a}}(-x)=\frac{1-(1+x)^{a}}{x}$, which can be written as

$$
\int_{1}^{\infty} \frac{1}{x+t} \cdot \frac{(t-1)^{a}}{t} d t=\frac{\pi a}{\sin (\pi a)} \cdot \frac{(1+x)^{a}-1}{a x}, \quad a \in(-1,0) .
$$

This identity extends to $a \in(-1,1)$ since the integral in the LHS exists and the both hands sides are real analytic functions of $a \in(-1,1)$. Let $\mu_{a}$ be the probability measure on $(1, \infty)$ with density $\frac{1}{B(1+a, 1 / 2-a)} \cdot \frac{(t-1)^{a}}{t^{3 / 2}}$ for $a \in(-1,1 / 2)$. From $(5.2)$ and (5.6), up to the multiplication of a constant the measure $\mu_{a} \circledast \mathbf{b}_{1 / 2,1}$ has the density $\frac{(1+x)^{a}-1}{a x^{3 / 2}}$.

We present the third example without a proof.

Example 5.3. Let $\mu$ be the beta distribution with density $\frac{1}{2 \sqrt{t}} 1_{(0,1)}(t) d t$. Then the measure $\mu \circledast \mathbf{b}_{1 / 2,1}$ is given by

$$
\frac{\log (1+1 / x)}{2 \pi \sqrt{x}} 1_{(0, \infty)}(x) d x .
$$

5.2. Limit Distributions of Multiplicative Free Laws of Large Numbers. Tucci investigated free multiplicative laws of large numbers for measures with compact support in [44] and then Haagerup and Möller proved the general case as follows [21]: If $\mu \in \mathcal{P}_{+}$, then the law

$$
\left(\mu^{\bigotimes n}\right)^{1 / n}
$$

weakly converges to a probability measure on $\mathbb{R}_{+}$, which we denote by $\Phi(\mu)$. A striking fact is that the limit law $\Phi(\mu)$ is not a delta measure unless $\mu$ is a delta measure. In fact the map $\Phi$ is even injective. The distribution function of this limit measure can be described in terms of the $S$-transform as follows:

$$
\Phi(\mu)(\{0\})=\mu(\{0\}), \quad \Phi(\mu)\left(\left[0, \frac{1}{S_{\mu}(x-1)}\right]\right)=x, \quad x \in(\mu(\{0\}), 1) .
$$

We compute $\Phi(\mu)$ when $\mu$ is a scale mixture of positive Boolean stable laws.

Theorem 5.4. Let $\mu \in \mathcal{P}_{+}$and $\alpha \in(0,1 / 2]$. 
(1) It holds that

$$
\Phi\left(\mu \otimes \mathbf{b}_{\alpha, 1}\right)=\left(\mu \otimes \mathbf{b}_{\frac{\alpha}{1-\alpha}, 1}\right) \circledast \mathbf{P a}(1),
$$

where $\mathbf{P a}(r)$ is the Pareto distribution

$$
\mathbf{P a}(r)(d x)=r(1+x)^{-r-1} 1_{(0, \infty)}(x) d x .
$$

In particular,

$$
\Phi\left(\mu \otimes \mathbf{b}_{1 / 2,1}\right)=\mu \circledast \mathbf{P a}(1) .
$$

(2) We have

$$
\Phi\left(\mu^{1 / \alpha} \circledast \mathbf{b}_{\alpha, 1}\right)=\left(\mu^{\bigotimes \frac{1}{1-\alpha}}\right)^{\frac{1-\alpha}{\alpha}} \circledast \mathbf{P a}(1) \circledast \mathbf{b}_{\frac{\alpha}{1-\alpha}, 1} .
$$

This implies that $\Phi\left(\mathcal{B}_{\alpha, 1}\right) \subset \mathcal{B}_{\frac{\alpha}{1-\alpha}, 1} \cap \mathcal{E} \mathcal{M}$ since $\mathbf{P a}(1) \in \mathcal{E} \mathcal{M}$. In particular, we have $\Phi\left(\mathcal{B}_{1 / 3,1}\right) \subset \mathcal{I D}(\boxplus) \cap \mathcal{E} \mathcal{M}$.

Proof. First we show (5.8) as follows:

$$
\begin{aligned}
\left(\left(\mu \bigotimes \mathbf{b}_{1 / 2,1}\right)^{\bigotimes n}\right)^{\frac{1}{1+n}} & =\left(\mu^{\bigotimes n} \bigotimes\left(\mathbf{b}_{1 / 2,1}\right)^{\bigotimes n}\right)^{\frac{1}{1+n}} \\
& \left.=\left(\mu^{\bigotimes \frac{n}{1+n}}\right)^{\bigotimes(1+n)} \bigotimes \mathbf{b}_{\frac{1}{1+n}, 1}\right)^{\frac{1}{1+n}} \\
& =\left(\left(\mu^{\bigotimes \frac{n}{1+n}}\right)^{1+n} \circledast \mathbf{b}_{\frac{1}{1+n}, 1}\right)^{\frac{1}{1+n}} \\
& \left.=\mu^{\bigotimes \frac{n}{1+n} \circledast\left(\mathbf{b}_{\frac{1}{1+n}}, 1\right.}\right)^{\frac{1}{1+n}},
\end{aligned}
$$

where we used (3.15) on the second line. If a measure $\nu \in \mathcal{P}_{+}$has a density $p(x)$, the measure $\nu^{q}$ has the density $\frac{1}{q} x^{\frac{1}{q}-1} p\left(x^{\frac{1}{q}}\right)$. It then follows that the density of $\left(\mathbf{b}_{q, 1}\right)^{q}$ is given by

$$
\frac{\sin (\pi q)}{\pi q} \cdot \frac{1}{x^{2}+2 x \cos (\pi q)+1},
$$

which converges to $(1+x)^{-2}$ uniformly on $[0, \infty)$ as $q \rightarrow 0$. Hence (5.8) has been proved.

Recall from (4.28) that the identity $\mathbf{b}_{1 / 2,1} \otimes \mathbf{b}_{\frac{\alpha}{1-\alpha}, 1}=\mathbf{b}_{\alpha, 1}$ holds. By replacing $\mu$ by $\mathbf{b}_{\frac{\alpha}{1-\alpha}, 1} \otimes \mu$ in (5.8), we obtain (5.7).

By replacing $\mu$ by $\mu^{\bigotimes 1 / \alpha}$ in (5.7), we have

$$
\begin{aligned}
\Phi\left(\mu^{1 / \alpha} \circledast \mathbf{b}_{\alpha, 1}\right) & =\Phi\left(\mu^{\bigotimes 1 / \alpha} \otimes \mathbf{b}_{\alpha, 1}\right) \\
& =\left(\left(\mu^{\bigotimes \frac{1}{1-\alpha}}\right)^{\bigotimes \frac{1-\alpha}{\alpha}} \otimes \mathbf{b}_{\frac{\alpha}{1-\alpha}, 1}\right) \circledast \mathbf{P a}(1) \\
& =\left(\left(\mu^{\bigotimes \frac{1}{1-\alpha}}\right)^{\frac{1-\alpha}{\alpha}} \circledast \mathbf{b}_{\frac{\alpha}{1-\alpha}, 1}\right) \circledast \mathbf{P a}(1) .
\end{aligned}
$$

Example 5.5. Theorem 5.4 in particular implies that $\Phi\left(\mathcal{B}_{\alpha, 1}\right) \subset \mathcal{I D}(\boxplus)$ for $\alpha \leq$ $1 / 3$. The constant $1 / 3$ is optimal as shown in the following example. Take $\mu$ to be the Boolean stable law $\mathbf{b}_{\alpha, 1}$ itself for $\alpha \in(0,1)$. Then

$$
S_{\mu}(z)=\Sigma_{\mu}\left(\frac{z}{1+z}\right)=\left(\frac{-z}{1+z}\right)^{\frac{1-\alpha}{\alpha}}=\left(\frac{-z}{1+z}\right)^{1 / \beta},
$$


where the new parameter $\beta=\frac{\alpha}{1-\alpha} \in(0, \infty)$ is introduced for simplicity. The compositional inverse function of $\frac{1}{S_{\mu}(x-1)}$ is now equal to $\frac{x^{\beta}}{1+x^{\beta}}$, and so we have $\Phi(\mu)([0, x])=\frac{x^{\beta}}{1+x^{\beta}}$ for $x \in[0, \infty)$. The density function is given by

$$
\frac{d \Phi(\mu)}{d x}(x)=\frac{\beta x^{\beta-1}}{\left(x^{\beta}+1\right)^{2}}, \quad x \in(0, \infty)
$$

which is a generalized beta distribution of second kind but a different one from Proposition 5.1. This measure is in $\mathcal{I D}(\boxplus) \cap \mathcal{E} \mathcal{M}$ for $\beta \in(0,1 / 2]$ from Theorem 5.4. From [23, Theorem 5.1], the measure $\Phi(\mu)$ is not in $\mathcal{I D}(\boxplus)$ for $\beta \in(1 / 2,2 / 3)$, and so the number $1 / 3$ is optimal.

5.3. Continuous Boolean Convolution. The continuous Boolean convolution $\mathbf{b}(\sigma)$ of Boolean stable laws is defined by

$$
\eta_{\mathbf{b}(\sigma)}(z)=-\int_{(0,1]}(-z)^{\alpha} \sigma(d \alpha), \quad z \in \mathbb{C}^{-},
$$

for nonnegative finite measure $\sigma$ supported on $(0,1]$ (see [2]). Symbolically this measure may be written as

$$
\mathbf{b}(\sigma)=\int_{(0,1]}^{\uplus} \mathbf{b}_{\alpha, 1} \sigma(d \alpha) .
$$

The density is given by

$$
\frac{1}{\pi} \cdot \frac{\int_{(0,1]} \sin (\alpha \pi) x^{1-\alpha} \sigma(d \alpha)}{\left(x+\int_{(0,1]} \cos (\alpha \pi) x^{1-\alpha} \sigma(d \alpha)\right)^{2}+\left(\int_{(0,1]} \sin (\alpha \pi) x^{1-\alpha} \sigma(d \alpha)\right)^{2}}, x>0 .
$$

Proposition 5.6. For nonnegative finite measure $\sigma$ on $(0,1 / 2]$, we have

$$
\mathbf{b}(\sigma)=\mathbf{b}\left(D_{2} \sigma\right)^{2} \circledast \mathbf{b}_{1 / 2,1} \in \mathcal{B}_{1 / 2,1} .
$$

Proof. We compare the $\eta$-transforms using Corollary 4.4:

$$
\begin{aligned}
\eta_{\mathbf{b}\left(D_{2} \sigma\right)^{2} \circledast \mathbf{b}_{1 / 2,1}}(z) & =\eta_{\mathbf{b}\left(D_{2} \sigma\right)}\left(-(-z)^{1 / 2}\right) \\
& =-\int_{0}^{1}(-z)^{\frac{1}{2} \alpha} D_{2} \sigma(d \alpha) \\
& =-\int_{0}^{1 / 2}(-z)^{\alpha} \sigma(d \alpha) \\
& =\eta_{\mathbf{b}(\sigma)}(z) .
\end{aligned}
$$

Example 5.7. A particularly interesting case comes when $\sigma:=\sum_{k=1}^{n}\left(\begin{array}{l}n \\ k\end{array}\right) \delta_{\frac{k}{n}} \alpha$. In this case we get

$$
\mathbf{b}(\sigma)=\left(\mathbf{m}_{1 / n, 1}\right)^{1 / \alpha} \circledast \mathbf{b}_{\alpha, 1} \in \mathcal{B}_{\alpha, 1} .
$$

This can be proved by computing the $\eta$-transform (see (3.10)). 
5.4. Probability Measures in $\mathcal{B}_{1, \rho} \backslash \mathcal{I} \mathcal{D}(\boxplus)$. We present a two-parameter family of probability measures, some of which belong to $\mathcal{B}_{1, \rho} \backslash \mathcal{I D}(\boxplus)$. This completes the proof of Theorem 4.25. For $t, \rho \in[0,1]$, let $\lambda_{t, \rho} \in \mathcal{B}_{1, \rho}$ be the probability measure

$$
\lambda_{t, \rho}:=\left((1-t) \delta_{0}+t \delta_{1}\right) \circledast \mathbf{c}_{\rho}=t \delta_{0}+\frac{1-t}{\pi} \cdot \frac{\sin \rho \pi}{(x+\cos \rho \pi)^{2}+\sin ^{2} \rho \pi} 1_{\mathbb{R}}(x) d x,
$$

which appeared in the proof of Theorem 4.18. The measures $\lambda_{0, t}$ and $\lambda_{1, t}$ are understood to be $t \delta_{0}+(1-t) \delta_{-1}$ and $t \delta_{0}+(1-t) \delta_{1}$ respectively.

Proposition 5.8. The measure $\lambda_{t, \rho}$ is FID if and only if: (1) $t=0$; (2) $t \in$ $[1 / 2,1],|\cos \rho \pi| \leq 2 t-1$. In particular, $\mathcal{B}_{1, \rho} \not \subset \mathcal{I D}(\boxplus)$.

Proof. Assume that $t, \rho \in(0,1)$; the other cases are Bernoulli distributions and are well known. The Cauchy transform of $\lambda_{t, \rho}$ is given by $G_{\lambda_{t, \rho}}(z)=\frac{t}{z}+\frac{1-t}{z+e^{i \rho \pi}}$, and so for some $\alpha, \beta>0$

$$
\phi_{\lambda_{t, \rho}}(z)=\frac{1}{2}\left(-z-e^{i \rho \pi}+\left(z^{2}+2(2 t-1) e^{i \rho \pi} z+e^{2 i \rho \pi}\right)_{(0,2 \pi)}^{1 / 2}\right), \quad z \in \Gamma_{\alpha, \beta} .
$$

Note that the polynomial $z^{2}+2(2 t-1) e^{i \rho \pi} z+e^{2 i \rho \pi}$ has the zeros $z_{ \pm}=e^{i(\rho \pi \pm \phi)}$, where $\cos \phi=1-2 t$ and $\phi \in(0, \pi)$. If $t \in(0,1 / 2)$, then $z_{+}$or $z_{-}$is contained in $\mathbb{C}^{+}$and so $\phi_{\lambda_{t, \rho}}$ does not extend to $\mathbb{C}^{+}$analytically, and so $\lambda_{t, \rho} \notin \mathcal{I D}(\boxplus)$ from Theorem 2.1. If $t \in[1 / 2,1)$, then both $z_{+}=e^{i(\rho \pi+\phi)}$ and $z_{-}=e^{i(\rho \pi-\phi)}$ are in $\mathbb{C}^{-} \cup \mathbb{R}$ if and only if $\pi-\phi \leq \rho \pi \leq \phi$ or equivalently $|\cos \rho \pi| \leq 2 t-1$.

Now suppose that $|\cos \rho \pi| \leq 2 t-1$. Then $\phi_{\lambda_{t, \rho}}$ extends analytically to $\mathbb{C}^{+}$and continuously to $\mathbb{C}^{+} \cup \mathbb{R}$, so from Theorem 2.1 we only have to show that

$$
\operatorname{Im}\left(\phi_{\lambda_{t, \rho}}(z)\right) \leq 0, \quad z \in \mathbb{C}^{+} \cup \mathbb{R} .
$$

First we are going to prove that $\operatorname{Im}\left(\phi_{\lambda_{t, \rho}}(x+i 0)\right) \leq 0$ for $x \in \mathbb{R}$. Let

$$
\begin{aligned}
r e^{i \theta}: & =x^{2}+2(2 t-1) e^{i \rho \pi} x+e^{2 i \rho \pi} \\
& =x^{2}+2(2 t-1)(\cos \rho \pi) x+\cos 2 \rho \pi+2 \sin \rho \pi(\cos \rho \pi+(2 t-1) x) i .
\end{aligned}
$$

The inequality (5.10) on $\mathbb{R}$ is equivalent to $\sqrt{r} \sin (\theta / 2) \leq \sin \rho \pi$ and from the formula $\sin ^{2}(\theta / 2)=(1-\cos \theta) / 2$, it is also equivalent to $r \leq r \cos \theta+2 \sin ^{2} \rho \pi=$ $x^{2}+2(2 t-1)(\cos \rho \pi) x+1$. The difference $\left(x^{2}+2(2 t-1)(\cos \rho \pi) x+1\right)^{2}-r^{2}$ turns out to be $16 t(1-t)\left(\sin ^{2} \rho \pi\right) x^{2} \geq 0$, showing the desired conclusion (5.10) for $z \in \mathbb{R}$.

Next, consider the bounded domain $D_{R}$ surrounded by the boundary $[-R, R] \cup$ $\left\{z \in \mathbb{C}^{+}:|z|=R\right\}$. We can easily show the estimate $\phi_{\lambda_{t, \rho}}(z)=-(1-t) e^{i \rho \pi}+o(1)$ uniformly as $z \rightarrow \infty, z \in \mathbb{C}^{+}$. Hence, (5.10) is valid on the boundary of $D_{R}$ for large $R>0$. From the maximum principle for (sub-) harmonic functions, the inequality (5.10) holds for any $z \in D_{R}$, and hence for $z \in \mathbb{C}^{+}$by taking the limit $R \rightarrow \infty$.

Moreover we can explicitly calculate the free divisibility indicator of $\lambda_{t, \rho}$.

Proposition 5.9. Let $t, \rho \in[0,1]$. Then

$$
\phi\left(\lambda_{t, \rho}\right)= \begin{cases}\frac{t}{1-t} \tan ^{2}\left(\frac{\rho \pi}{2}\right), & t \in(0,1), \rho \in[0,1 / 2], \\ \frac{t}{1-t} \tan ^{2}\left(\frac{(1-\rho) \pi}{2}\right), & t \in(0,1), \rho \in[1 / 2,1], \\ \infty, & t \in\{0,1\} .\end{cases}
$$


Proof. We assume $t \in(0,1)$; otherwise $\lambda_{t, \rho}$ is a delta measure or a Cauchy distribution whose free divisibility indicator is infinity. By computing $\eta$-transforms, we get

$$
\left(\lambda_{t, \rho}\right)^{\uplus u}=D_{(1-t) u+t}\left(\lambda_{\frac{t}{(1-t) u+t}, \rho}\right), \quad u>0 .
$$

From Proposition 5.8, this is FID if and only if $|\cos \rho \pi| \leq \frac{2 t}{(1-t) u+t}-1$. From (2.11), $\phi\left(\lambda_{t, \rho}\right)$ is the solution $u$ of the equation $|\cos \rho \pi|=\frac{2 t}{(1-t) u+t}-1$, giving the assertion of the proposition.

5.5. Free Jurek Class and $\mathcal{B}_{\alpha, \rho}$. The second-named author and Thorbjørnsen established the free analogue of Yamazato's theorem, saying that any freely selfdecomposable distribution is unimodal [27]. We want to find examples of freely selfdecomposable distributions from measures in $\mathcal{B}_{\alpha, \rho}$, but such is not possible at least for positive and symmetric cases. This is because scale mixtures of Boolean stable laws are compound free Poisson distributions (see Proposition 4.21), but a nontrivial freely selfdecomposable distribution does not have a finite Lévy measure. Instead, we will consider a class called the free Jurek class that is larger than the class of freely selfdecomposable distributions. We consider scale mixtures of Boolean stable laws which belong to the free Jurek class.

The classical Jurek class was studied in [31] and it coincides with all the distributions of stochastic integrals of the form $\int_{0}^{1} t d X_{t}$, where $\left(X_{t}\right)_{t \geq 0}$ is a Lévy process starting at 0 .

Definition 5.10. An FID distribution $\mu$ is said to be freely s-selfdecomposable if the Lévy measure $\nu_{\mu}$ is unimodal with mode 0 . The set $\mathcal{U}(\boxplus)$ of all freely sselfdecomposable distributions is called the free Jurek class.

We quote a special case of [26, Theorem 4].

Lemma 5.11. (1) The positive Boolean stable law $\mathbf{b}_{\alpha, 1}$ is unimodal with mode 0 if and only if $\alpha \in\left(0, \alpha_{0}\right]$, where $\alpha_{0}=0.7364 \ldots$ is the unique solution $x \in(0,1)$ of the equation $\sin \pi x=x$.

(2) The symmetric Boolean stable law $\mathbf{b}_{\alpha, 1 / 2}$ is unimodal with mode 0 if and only if $\alpha \in(0,1]$.

The following result follows from Khintchine's characterization of unimodality (see [32] or [49, Theorem 2.7.3]): a probability measure $\mu$ is unimodal with mode 0 if and only if $\mu=\mathbf{u} \circledast \rho$ for some $\rho \in \mathcal{P}$, where $\mathbf{u}$ is the uniform distribution on $[0,1]$.

Lemma 5.12. If $\mu \in \mathcal{P}$ is unimodal with mode 0 , then so is $\nu \circledast \mu$ for any $\nu \in \mathcal{P}$.

Theorem 5.13. Let $\alpha_{1}=\alpha_{0} /\left(1+\alpha_{0}\right)=0.4241 \ldots$, where $\alpha_{0}$ is the number defined in Lemma 5.11. The following statements hold.

(1) $\mathcal{B}_{\alpha, 1} \subset \mathcal{U}(\boxplus)$ for $\alpha \in\left(0, \alpha_{1}\right]$

(2) $\mathcal{B}_{\alpha, 1 / 2} \subset \mathcal{U}(\boxplus)$ for $\alpha \in(0,1 / 2]$.

Proof. Let $\mu \in \mathcal{P}_{+}, \beta=\alpha /(1-\alpha)$ and assume that $\rho=1 / 2$ or 1 . Note first that $\mu^{1 / \alpha} \circledast \mathbf{b}_{\alpha, \rho}$ is FID if $\alpha \leq \alpha_{1}<1 / 2, \rho=1$ or if $\alpha \leq 1 / 2<2 / 3, \rho=1 / 2$ from Theorem 4.25. The computation of $S$-transforms gives us the formula

$$
\mathbf{b}_{\alpha, \rho}=\boldsymbol{\pi} \otimes\left(\mathbf{f}_{1-\alpha, 1}\right)^{\otimes 1 / \beta} \otimes \mathbf{b}_{\beta, \rho} .
$$


Then we have

$$
\begin{aligned}
& \mu^{1 / \alpha} \circledast \mathbf{b}_{\alpha, \rho}=\mu^{\bigotimes 1 / \alpha} \otimes \mathbf{b}_{\alpha, \rho} \\
& =\mu^{\bigotimes 1 / \alpha} \otimes \boldsymbol{\pi} \otimes\left(\mathbf{f}_{1-\alpha, 1}\right)^{\bigotimes 1 / \beta} \otimes \mathbf{b}_{\beta, \rho} \\
& =\boldsymbol{\pi} \otimes\left(\left(\mu^{\bigotimes \frac{1}{1-\alpha}} \otimes \mathbf{f}_{1-\alpha, 1}\right)^{\bigotimes 1 / \beta} \otimes \mathbf{b}_{\beta, \rho}\right) \\
& =\boldsymbol{\pi} \otimes\left(\left(\mu^{\otimes \frac{1}{1-\alpha}} \otimes \mathbf{f}_{1-\alpha, 1}\right)^{1 / \beta} \circledast \mathbf{b}_{\beta, \rho}\right),
\end{aligned}
$$

where we used (4.9) on the first and last lines. This means that the Lévy measure of $\mu^{1 / \alpha} \circledast \mathbf{b}_{\alpha, \rho}$ is given by the probability measure

$$
\left(\mu^{\otimes \frac{1}{1-\alpha}} \otimes \mathbf{f}_{1-\alpha, 1}\right)^{1 / \beta} \circledast \mathbf{b}_{\beta, \rho} ;
$$

see Remark 2.2. If $\alpha \leq \alpha_{1}$ and $\rho=1$, then $\beta \leq \alpha_{0}$ and $\mathbf{b}_{\beta, 1}$ is unimodal with mode 0 from Lemma 5.11, and so is the Lévy measure from Lemma 5.12. The symmetric case $\rho=1 / 2$ is similar.

Remark 5.14. (1) As usual, we cannot use the $S$-transform for general $\rho$, and we cannot extend Theorem 5.13 for $\rho \neq 0,1 / 2,1$.

(2) The Lévy measure (5.13) can be written as

$$
\mathbf{B}_{\alpha /(1-\alpha), \rho}\left(\mathbf{F}_{1-\alpha, 1}(\mu)\right)
$$

with notations in Theorem 4.14 .

\section{Existence of Free Bessel Laws}

In this section, we prove Theorem 1.2 which also settles the problem of definition of free Bessel laws stated in Banica et al. [8].

6.1. Free Powers of Free Poisson. Given $\mu \in \mathcal{P}_{+}$, one can ask whether the convolution powers $\mu^{\bigotimes s}$ and $\mu^{\boxplus t}$ exist for various values of $s, t>0$. Specifically, the question is whether $S_{\mu}(z)^{s}$ and $t \mathcal{C}_{\mu}^{\boxplus}(z)$ are the $S$ - and free cumulant transforms of some probability measures. It is known that for $s>1$ or $t>1$, the convolution powers $\mu^{\boxplus s}$ and $\mu^{\boxplus t}$ always exist as probability measures.

Furthermore, one can ask whether the convolution powers $\left(\mu^{\bigotimes_{s}}\right)^{\boxplus t}$ or $\left(\mu^{\boxplus t}\right)^{\rrbracket_{s}}$ exist, for different values of $s, t>0$. Since we have the following "commutation" relation,

$$
\left(\mu^{\boxplus t}\right)^{\unrhd s}=D_{t^{s-1}}\left(\mu^{\boxplus s}\right)^{\boxplus t},
$$

for $t \geq 1$ and $s \geq 1$ then both questions are equivalent.

We answer this question for the case when $\mu=\pi$. Since the free Poisson distribution $\boldsymbol{\pi}$ is $\otimes$-infinitely divisible and free regular (the latter meaning that $\boldsymbol{\pi}^{\boxplus t} \in \mathcal{P}_{+}$ for any $t>0$; see [5]), then the double power $\tilde{\boldsymbol{\pi}}_{s t}=\left(\boldsymbol{\pi}^{\boxplus s}\right)^{\boxplus t}$ exists as a probability measure when $\max (s, t) \geq 1$.

The moments and cumulants of $\tilde{\boldsymbol{\pi}}_{s t}$ were studied by Hinz and Młotkowski [28]. In particular, they state the following conjecture which is closely related to the question of the possible parameters of free Bessel laws in Banica et al. [8] as we explain below. 
Conjecture 6.1 $([28]) . \quad \tilde{\boldsymbol{\pi}}_{s t}$ is a probability measure if and only if $\max (s, t) \geq 1$. Equivalently, the sequence given by $\tilde{m}_{0}(s, t)=1$ and

$$
\tilde{m}_{n}(s, t)=\sum_{k=1}^{n} \frac{t^{k}}{n}\left(\begin{array}{c}
n \\
k-1
\end{array}\right)\left(\begin{array}{c}
n s \\
n-k
\end{array}\right)
$$

is positive definite if and only if $\max (s, t) \geq 1$.

We solve the conjecture of Hinz and Młotkowski in the affirmative. We will use the relation between free Poisson, free stable and Boolean stable laws

$$
\mathbf{b}_{\alpha, 1}=\boldsymbol{\pi}^{\otimes \frac{1-\alpha}{\alpha}} \otimes \mathbf{f}_{\alpha, 1}
$$

proved in (3.17) together with the following lemma.

Lemma 6.2. For $1 / 2<\alpha<1$ and $t<1,\left(\mathbf{b}_{\alpha, 1}\right)^{\boxplus t}$ does not exist as a probability measure.

Proof. The Boolean stable law $\mathbf{b}_{\alpha, 1}$ is not FID for $\alpha \in(1 / 2,1)$ [2], and so $\phi\left(\mathbf{b}_{\alpha, 1}\right)<$ 1 from Theorem 2.3(2). From the arguments in Subsection 3.3 of [4], the free divisibility indicator $\phi\left(\mathbf{b}_{\alpha, 1}\right)$ is either 0 or $\infty$, but since it is smaller than 1 , it is 0 . Then Theorem 2.3(1) implies the conclusion.

Now, we are in position to prove the first part of Theorem 1.2. That is, we prove the conjecture by Hinz and Młotkowski.

Proof of Conjecture 6.1. Let $0<s, t<1, \alpha=\frac{1}{1+s}$ and $K=t^{1-1 / \alpha}$. Suppose that $\tilde{\boldsymbol{\pi}}_{s t}$ is a probability measure. Then so is $b(s, t):=\tilde{\boldsymbol{\pi}}_{s t} \otimes \mathbf{f}_{\alpha, 1}$. Since $t<1$, we can take the $1 / t$ free additive power, yielding

$$
\begin{aligned}
b(s, t)^{\boxplus 1 / t} & =\left(\tilde{\boldsymbol{\pi}}_{s t} \otimes \mathbf{f}_{\alpha, 1}\right)^{\boxplus 1 / t} \\
& =D_{t}\left(\left(\boldsymbol{\pi}^{\bigotimes s}\right)^{\boxplus t}\right)^{\boxplus 1 / t} \otimes\left(\mathbf{f}_{\alpha, 1}\right)^{\boxplus 1 / t} \\
& =D_{t}\left(\boldsymbol{\pi}^{\boxplus s}\right) \otimes D_{t^{-1 / \alpha}}\left(\mathbf{f}_{\alpha, 1}\right) \\
& =D_{K}\left(\boldsymbol{\pi}^{\boxplus s} \otimes \mathbf{f}_{\alpha, 1}\right),
\end{aligned}
$$

where we used (2.4) in the second equality and the stability property of $\mathbf{f}_{\alpha, 1}$ in the third. Since $\alpha=\frac{1}{s+1}$, then $s=(1-\alpha) / \alpha$ and $s \in(0,1)$. Thus, we have proved that, for $1 / 2<\alpha<1$,

$$
b(s, t)^{\boxplus 1 / t}=D_{K}\left(\boldsymbol{\pi}^{\otimes \frac{1-\alpha}{\alpha}} \otimes \mathbf{f}_{\alpha, 1}\right)=D_{K}\left(\mathbf{b}_{\alpha, 1}\right) .
$$

This means $\left(\mathbf{b}_{\alpha, 1}\right)^{\boxplus t}$ exists as a probability measure but this is impossible by Lemma 6.2 .

6.2. Free Bessel Laws. Let us recall the definition of free Bessel laws, together with some basic facts. The free Bessel laws were introduced in Banica et al. [8] as a two-parameter family of probability measures on $\mathbb{R}_{+}$generalizing the free Poisson $\boldsymbol{\pi}$. They studied connections with random matrices, quantum groups and $k$-divisible non-crossing partitions.

The original definition of the free Bessel law is the following.

Definition 6.3. The free Bessel law is the probability measure $\boldsymbol{\pi}_{s t}$ with $(s, t) \in$ $(0, \infty) \times(0, \infty)-(0,1) \times(1, \infty)$, defined as follows:

(1) For $s \geq 1$ we set $\boldsymbol{\pi}_{s t}=\boldsymbol{\pi}^{\bigotimes(s-1)} \otimes \boldsymbol{\pi}^{\boxplus t}$; 
(2) For $t \leq 1$ we set $\boldsymbol{\pi}_{s t}=\left((1-t) \delta_{0}+t \delta_{1}\right) \otimes \boldsymbol{\pi}^{\otimes s}$.

The compatibility between (1) and (2) comes from the following identity valid for $s \geq 1$ and $0<t \leq 1$ :

$$
\boldsymbol{\pi}^{\otimes(s-1)} \otimes \boldsymbol{\pi}^{\boxplus t}=\left((1-t) \delta_{0}+t \delta_{1}\right) \otimes \boldsymbol{\pi}^{\bigotimes s} .
$$

Special important cases are $t=1$ for which $\pi_{s 1}=\pi^{\bigotimes s}$ and $s=1$ for which $\boldsymbol{\pi}_{1 t}=\boldsymbol{\pi}^{\boxplus t}$

The moments of free Bessel law $\boldsymbol{\pi}_{s t}$ are calculated as follows [8]:

$$
m_{n}(s, t)=\sum_{k=1}^{n} \frac{t^{k}}{k}\left(\begin{array}{l}
n-1 \\
k-1
\end{array}\right)\left(\begin{array}{c}
n s \\
k-1
\end{array}\right) .
$$

In the particular case where $t=1$ and $s$ is an integer we obtain the Fuss-Catalan numbers $m_{n}=\frac{1}{s n+1}\left(\begin{array}{c}s n+n \\ n\end{array}\right)$, known to appear in several contexts. In particular, they count the number of $s$-divisible and $(s+1)$-equal non-crossing partitions. For details on $s$-divisible non-crossing partitions, see Edelman [19], Stanley [42], Arizmendi [1] and Armstrong [7].

Banica et al. [8] considered the question of whether $\boldsymbol{\pi}_{s t}$ exists as a probability measure for certain points in the critical rectangle $(0,1) \times(1, \infty)$. The precise range of the parameters $(s, t)$ was an open problem. We can determine it from Conjecture 6.1. Indeed, one recognizes the moments in (6.2) as the moments of $\tilde{\boldsymbol{\pi}}_{s, 1 / t}$ multiplied by $t^{n+1}$, and thus we get the following (or see the proof of $[8$, Theorem 3.1]).

Lemma 6.4. Let $s, t>0$. We have

$$
\boldsymbol{\pi}_{s t}=(1-t) \delta_{0}+t D_{t}\left(\tilde{\boldsymbol{\pi}}_{s, 1 / t}\right),
$$

or equivalently

$$
\tilde{\boldsymbol{\pi}}_{s t}=(1-t) \delta_{0}+t D_{t}\left(\boldsymbol{\pi}_{s, 1 / t}\right),
$$

where equalities are in the sense of linear functionals on the polynomial ring $\mathbb{C}[x]$, e.g. $\boldsymbol{\pi}_{s t}\left(x^{n}\right)=m_{n}(s, t), n \geq 0$.

From the previous lemma we directly get the following, proving the last part of Theorem 1.2.

Corollary 6.5. $\boldsymbol{\pi}_{s t}$ is not a probability measure for $t>1$ and $s<1$.

Proof. Suppose $\boldsymbol{\pi}_{s, 1 / t}$ is a probability measure for some $0<s, t<1$. Then from (6.4), $\tilde{\boldsymbol{\pi}}_{s t}$ is a probability measure too. This is a contradiction to Conjecture 6.1 which we proved to be true.

\section{REFERENCES}

[1] O. Arizmendi, Statistics of blocks in k-divisible non-crossing partitions, Electron. J. Combin. 19 (2012), no. 2, Paper 47, 22 pp.

[2] O. Arizmendi and T. Hasebe, Classical and free infinite divisibility for Boolean stable laws, Proc. Amer. Math. Soc. 142 (2014), no. 5, 1621-1632.

[3] O. Arizmendi and T. Hasebe, On a class of explicit Cauchy-Stieltjes transforms related to monotone stable and free Poisson laws, Bernoulli 19 (2013), no. 5B, 2750-2767.

[4] O. Arizmendi and T. Hasebe, Semigroups related to additive and multiplicative, free and Boolean convolutions, Studia Math. 215 (2013), no. 2, 157-185.

[5] O. Arizmendi, T. Hasebe and N. Sakuma, On the law of free subordinators, ALEA Lat. Am. J. Probab. Math. Stat. 10 (2013), no. 1, 271-291. 
[6] O. Arizmendi and V. Pérez-Abreu, The S-transform for symmetric probability measures with unbounded supports, Proc. Amer. Math. Soc. 137 (2009), 3057-3066.

[7] D. Armstrong, Generalized noncrossing partitions and combinatorics of Coxeter groups, Mem. Amer. Math. Soc. 202 (2009), no. 949, x+159 pp.

[8] T. Banica, S.T. Belinschi, M. Capitane and B. Collins, Free Bessel laws, Canad. J. Math. 63 (2011), no. 1, 3-37.

[9] O.E. Barndorff-Nielsen and S. Thorbjørnsen, Lévy laws in free probability, Proc. Nat. Acad. Sci. 99 (2002), 16568-16575.

[10] O.E. Barndorff-Nielsen and S. Thorbjørnsen, Self-decomposability and Lévy processes in free probability, Bernoulli 8(3) (2002), 323-366.

[11] S.T. Belinschi and H. Bercovici, Partially defined semigroups relative to multiplicative free convolution, Int. Math. Res. Notices, No. 2 (2005), 65-101.

[12] S.T. Belinschi, M. Bożejko, F. Lehner and R. Speicher, The normal distribution is $\boxplus-$ infinitely divisible, Adv. Math. 226, No. 4 (2011), 3677-3698.

[13] S.T. Belinschi and A. Nica, On a remarkable semigroup of homomorphisms with respect to free multiplicative convolution, Indiana Univ. Math. J. 57, No. 4 (2008), 1679-1713.

[14] H. Bercovici, Multiplicative monotonic convolution, Illinois J. Math. 49, No. 3 (2005), 929951.

[15] H. Bercovici and V. Pata, Stable laws and domains of attraction in free probability theory (with an appendix by Philippe Biane), Ann. of Math. (2) 149, No. 3 (1999), 1023-1060.

[16] H. Bercovici and D. Voiculescu, Free convolution of measures with unbounded support, Indiana Univ. Math. J. 42, No. 3 (1993), 733-773.

[17] L. Bondesson, Generalized gamma convolutions and related classes of distributions and densities, Lecture Notes in Stat. 76, Springer, New York, 1992.

[18] M. Bożejko and T. Hasebe, On free infinite divisibility for classical Meixner distributions. arXiv: 1302.4885

[19] P.H. Edelman, Chain enumeration and noncrossing partitions, Discrete Math. 31 (1980), $171-180$.

[20] U. Franz, Monotone and boolean convolutions for non-compactly supported probability measures, Indiana Univ. Math. J. 58, No. 3 (2009), 1151-1186.

[21] U. Haagerup and S. Möller, The law of large numbers for the free multiplicative convolution, in Operator Algebra and Dynamics, volume 58 of Springer Proceedings in Mathematics \& Statistics, 157-186, Springer, Berlin/Heidelberg, 2013.

[22] U. Haagerup and H. Schultz, Brown measures of unbounded operators affiliated with a finite von Neumann algebra, Math. Scand. 100 (2007), 209-263.

[23] T. Hasebe, Free infinite divisibility for beta distributions and related ones. arXiv:1305.0924

[24] T. Hasebe, Monotone convolution and monotone infinite divisibility from complex analytic viewpoints, Infin. Dimens. Anal. Quantum Probab. Relat. Top. 13, No. 1 (2010), 111-131.

[25] T. Hasebe and A. Kuznetsov, On free stable distributions. arXiv:1404.2981

[26] T. Hasebe and N. Sakuma, Unimodality of Boolean and monotone stable distributions. arXiv: 1403.2487

[27] T. Hasebe and S. Thorbjørnsen, Unimodality of the freely selfdecomposable probability laws. arXiv:1309:6776

[28] M. Hinz and W. Młotkowski, Free powers of the free Poisson measure, Colloq. Math. 123 (2011), no. 2, 285-290.

[29] H.-W. Huang, Supports, regularity and $\boxplus$-infinite divisibility for measures of the form $\left(\mu^{\boxplus p}\right)^{\uplus q}$. arXiv: $1209.5787 \mathrm{v} 1$

[30] W. Jedidi and T. Simon, Further examples of GGC and HCM densities, Bernoulli 19 (2013), no. 5A, 1818-1838.

[31] Z.J. Jurek, Relations between the s-selfdecomposable and selfdecomposable measures, Ann. Probab. 13 (1985), No. 2, 592-608.

[32] A. Ya. Khintchine, On unimodal distributions, Izv. Nauk Mat. i Mekh. Inst. Tomsk 2 (1938), 1-7 (in Russian)

[33] H. Maassen, Addition of freely independent random variables, J. Funct. Anal. 106 (1992), 409-438.

[34] N. Muraki, The five independences as natural products, Infin. Dimens. Anal. Quantum Probab. Relat. Top. 6 (2003), 337-371. 
[35] A. Nica and R. Speicher, A "Fourier transform" for multiplicative functions on non-crossing partitions, J. Algebraic Combin. 6 (1997), no. 2, 141-160.

[36] A. Nica and R. Speicher, Lectures on the Combinatorics of Free Probability, London Mathematical Society Lecture Notes Series 335, Cambridge University Press, Cambridge, 2006.

[37] A. Nica and R. Speicher, On the multiplication of free N-tuples of noncommutative random variables, Amer. J. Math. 118 (1996), No. 4, 799-837.

[38] N. Raj Rao and R. Speicher, Multiplication of free random variables and the S-transform: The case of vanishing mean, Elect. Comm. Probab. 12 (2007), 248-258.

[39] K. Sato, Lévy Processes and Infinitely Divisible Distributions, Cambridge studies in advanced math. 68, 1999.

[40] R. Speicher, Combinatorial theory of the free product with amalgamation and operatorvalued free probability theory, Memoirs of the American Mathematical Society 132 (1998), no. 627 .

[41] R. Speicher and R. Woroudi, Boolean convolution, in Free Probability Theory, Ed. D. Voiculescu, Fields Inst. Commun. 12 (Amer. Math. Soc., 1997), 267-280.

[42] R.P. Stanley, Parking functions and noncrossing partitions, Electron. J. Combin. 4 (1997), $1-14$.

[43] F.W. Steutel, K. van Harn, Infinite Divisibility of Probability Distributions on the Real Line, Marcel Dekker, NewYork, 2004.

[44] G. Tucci, Limits laws for geometric means of free random variables, Indiana Univ. Math. J. 59 (2010), no. 1, 1-13.

[45] D. Voiculescu, Symmetries of some reduced free product $C^{*}$-algebras, in: Operator Algebras and their Connections with Topology and Ergodic Theory, 556-588, Lecture Notes in Mathematics, Vol. 1132, Springer-Verlag, Berlin/New York, 1985.

[46] D. Voiculescu, Addition of certain non-commutative random variables, J. Funct. Anal. 66 (1986), 323-346.

[47] D. Voiculescu, Multiplication of certain noncommuting random variables, J. Operator Theory 18 (1987), 223-235.

[48] J.-C. Wang, Strict limit types for monotone convolution, J. Funct. Anal. 262, no. 1 (2012), $35-58$.

[49] V.M. Zolotarev, One-dimensional stable distributions, volume 65 of Translations of Mathematical Monographs, American Mathematical Society, Providence, RI, 1986.

Department of Probability and Statistics, Cimat, Guanajuato, Mexico

E-mail address: octavius@cimat.mx

Department of Mathematics, Hokkaido University, Kita 10, Nishi 8, Kitaku, Sapporo 060-0810, JAPAN

E-mail address: thasebe@math.sci.hokudai.ac.jp 